\title{
Homotopy invariance through small stabilizations
}

\author{
Beatriz Abadie - Guillermo Cortiñas
}

Received: 12 April 2013 / Accepted: 20 November 2013 / Published online: 10 December 2013

(C) Tbilisi Centre for Mathematical Sciences 2013

\begin{abstract}
We associate an algebra $\Gamma^{\infty}(\mathfrak{A})$ to each bornological algebra $\mathfrak{A}$. Each symmetric ideal $S$ of the algebra $\ell^{\infty}$ of complex bounded sequences gives rise to an ideal $I_{S(\mathfrak{A})}$ of $\Gamma^{\infty}(\mathfrak{A})$. We show that all ideals arise in this way when $\mathfrak{A}$ is the algebra of complex numbers. We prove that for suitable $S$, Weibel's $K$-theory of $I_{S(\mathfrak{A})}$ is homotopy invariant, and show that the failure of the map from Quillen's to Weibel's $K$-theory of $I_{S(\mathfrak{A})}$ to be an isomorphism is measured by cyclic homology.
\end{abstract}

Keywords Operator ideal · Calkin's theorem - Crossed product · Karoubi's cone · K-theory

\section{Introduction}

Let $\ell^{2}=\ell^{2}(\mathbb{N})$ be the Hilbert space of square-summable sequences of complex numbers and $\mathcal{B}=\mathcal{B}\left(\ell^{2}\right)$ the algebra of bounded operators. Let Emb be the inverse monoid of all partially defined injections

\section{Communicated by Ralf Meyer.}

Both authors were supported by MathAmSud network U11MATH-05, partially funded by ANII, Uruguay, and by MINCyT, Argentina. Cortiñas was partially supported by CONICET and by grants UBACyT W386, PIP 112-200801-00900 and MTM2007-64704 (FEDER funds).

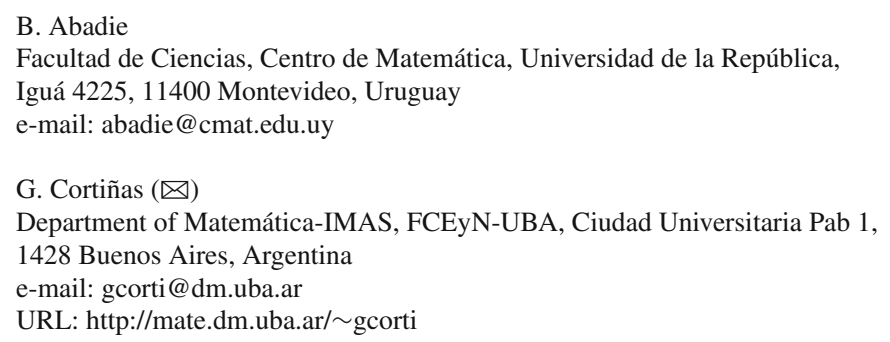




$$
\mathbb{N} \supset \operatorname{dom} f \stackrel{f}{\longrightarrow} \mathbb{N} \text {. }
$$

Each element $f \in$ Emb defines a partial isometry $U_{f} \in \mathcal{B}$; for the canonical Hilbert basis we have $U_{f}\left(e_{n}\right)=e_{f(n)}$ if $n \in \operatorname{dom} f$ and 0 otherwise. Similarly, each bounded sequence of complex numbers $\alpha \in \ell^{\infty}$ defines an element $\operatorname{diag}(\alpha) \in \mathcal{B}$ by $\operatorname{diag}(\alpha)\left(e_{n}\right)=\alpha(n) e_{n}$. The subspace generated by all the $U_{f}$ and $\operatorname{diag}(\alpha)$ with $f \in$ Emb and $\alpha \in \ell^{\infty}$ is the subalgebra

$$
\mathcal{B} \supset \Gamma^{\infty}:=\operatorname{span}\left\{\operatorname{diag}(\alpha) U_{f}: \alpha \in \ell^{\infty}, f \in \operatorname{Emb}\right\} .
$$

In this article we show that the algebra $\Gamma^{\infty}$ has several remarkable properties. One of them is that the lattice of two-sided ideals of $\Gamma^{\infty}$ is isomorphic to the lattice of twosided ideals of $\mathcal{B}$. A theorem of Calkin [2], as restated by Garling [16], establishes a one-to-one correspondence between two-sided ideals of $\mathcal{B}$ and the ideals of $\ell^{\infty}$ that are symmetric, that is, invariant under the action of Emb. Calkin's correspondence maps a symmetric ideal $S \triangleleft \ell^{\infty}$ to the ideal $J_{S}$ of those operators whose sequence of singular values belongs to $S$. Consider the subspace

$$
\Gamma^{\infty} \supset I_{S}:=\operatorname{span}\left\{\operatorname{diag}(\alpha) U_{f}: \alpha \in S, f \in \mathrm{Emb}\right\} .
$$

Note that $I_{\ell^{\infty}}=\Gamma^{\infty}$; for all symmetric ideals $S, I_{S} \triangleleft \Gamma^{\infty}$ is a two-sided ideal. We prove (see Theorem 4.2)

Theorem 1.1 The map $J \mapsto J \cap \Gamma^{\infty}$ is a bijection between the sets of two-sided ideals of $\mathcal{B}\left(\ell^{2}(\mathbb{N})\right)$ and $\Gamma^{\infty}$. If $S \triangleleft \ell^{\infty}$ is a symmetric ideal, then $J_{S} \cap \Gamma^{\infty}=I_{S}$.

More generally, we define for any bornological algebra $\mathfrak{A}$ (in particular for a Banach algebra $\mathfrak{A})$ an algebra $\Gamma^{\infty}(\mathfrak{A})$. The algebra $\Gamma^{\infty}(\mathfrak{A})$ contains an ideal $I_{S(\mathfrak{A})}$ for any symmetric ideal $S \triangleleft \ell^{\infty}$, and $S \mapsto I_{S(\mathfrak{A})}$ is a lattice homomorphism. Thus the smallest nonzero $I_{S(\mathfrak{A})}$ occurs when $S$ is the symmetric ideal $c_{f} \triangleleft \ell^{\infty}$ of finitely supported sequences; we get

$$
I_{c_{f}(\mathfrak{A})}=M_{\infty} \mathfrak{A}=\bigcup_{n} M_{n} \mathfrak{A} .
$$

Hence the inclusion $\mathfrak{A} \rightarrow M_{\infty} \mathfrak{A}$ into the upper left corner gives a stability homomorphism

$$
\iota_{S}: \mathfrak{A} \rightarrow I_{c_{f}(\mathfrak{A})} \subset I_{S(\mathfrak{A})}
$$

If $\mathfrak{A}$ is unital then $\iota_{c_{f}}$ induces an isomorphism in algebraic $K$-theory, by matrix stability. At the other extreme, $I_{\ell}(\mathfrak{A})=\Gamma^{\infty}(\mathfrak{A})$ is a ring with infinite sums in the sense of [22] (see Proposition 5.1); this permits the Eilenberg swindle and we have

$$
K_{*}\left(\Gamma^{\infty}(\mathfrak{A})\right)=0 .
$$

For $c_{f} \subsetneq S \subsetneq \ell^{\infty}$, the $K$-theory of $I_{S(\mathfrak{A})}$ is more interesting. We study it for

$$
S \in\left\{c_{0}, \ell^{p-}, \ell^{q}, \ell^{q+} \quad(p \leq \infty, q<\infty)\right\} .
$$


Here $c_{0}$ is the ideal of sequences vanishing at infinity, $\ell^{q}$ consists of the $q$-summable sequences, and

$$
\ell^{p-}=\bigcup_{r<p} \ell^{r}, \ell^{q+}=\bigcap_{s>q} \ell^{s} .
$$

Let BAlg be the category of bornological algebras. We consider several variants of $K$-theory. We write $K$ for algebraic $K$-theory, $K H$ for Weibel's homotopy algebraic $K$-theory and $K^{\text {top }}$ for topological $K$-theory. A bornological algebra is a bornolocal $C^{*}$-algebra if it is a filtering union of $C^{*}$-algebras. The following result follows from Theorem 8.2.

Theorem 1.2 i) The functor BAlg $\rightarrow \mathfrak{A} \mathfrak{b}, \mathfrak{A} \mapsto K H_{*}\left(I_{c_{0}(\mathfrak{A})}\right)$ is invariant under continuous homotopy.

ii) If $\mathfrak{A}$ is a bornolocal $C^{*}$-algebra and $n \geq 0$, then there is a natural split monomorphism

$$
K_{n}^{\text {top }}(\mathfrak{A})>-K H_{n}\left(I_{c_{0}(\mathfrak{A})}\right) .
$$

iii) If $n \leq 0$, then the comparison map

$$
K_{n}\left(I_{c_{0}(\mathfrak{A})}\right) \rightarrow K H_{n}\left(I_{c_{0}(\mathfrak{A})}\right)
$$

is an isomorphism for every $\mathfrak{A} \in \mathrm{BAlg}$.

The results above should be compared with Karoubi's conjecture (Suslin-Wodzicki's theorem [21, Theorem 10.9]) that for a $C^{*}$-algebra $\mathfrak{A}$, the comparison map

$$
K_{*}(\mathfrak{A} \tilde{\otimes} \mathcal{K}) \rightarrow K_{*}^{\text {top }}(\mathfrak{A} \tilde{\otimes} \mathcal{K}) \cong K_{*}^{\text {top }}(\mathfrak{A})
$$

is an isomorphism. Hence we may think of $\mathfrak{A} \rightarrow I_{c_{0}(\mathfrak{A})}$ as a smaller version of the stabilization $\mathfrak{A} \mapsto \mathfrak{A} \otimes \mathcal{K}$ whose homotopy algebraic $K$-theory is continuously homotopy invariant and contains $K_{*}^{\text {top }}(\mathfrak{A})$ as a direct summand. Next let $p \geq 1$ and consider the Schatten ideal $\mathcal{L}^{p} \triangleleft \mathcal{B}$. Notice that $\mathcal{L}^{p}$ is the ideal corresponding to $\ell^{p}$ under Calkin's correspondence. We have

$$
\mathcal{L}^{p}=J_{\ell p}
$$

Recall from [9, Theorem 6.2.1] that if $\mathfrak{A}$ is a locally convex algebra and $\mathfrak{A} \hat{\otimes} \mathcal{L}^{p}$ is the projective tensor product then

$$
K H_{*}\left(\mathfrak{A} \hat{\otimes} \mathcal{L}^{1}\right) \stackrel{\cong}{\longrightarrow} K H_{*}\left(\mathfrak{A} \hat{\otimes} \mathcal{L}^{p}\right) \stackrel{\cong}{\longrightarrow} K_{*}^{\text {top }}\left(\mathfrak{A} \hat{\otimes} \mathcal{L}^{p}\right)
$$

In the present article (Theorem 8.1) we prove the following analogue of the latter result. 
Theorem 1.3 Let $S$ be one of $\ell^{p}, \ell^{p+}(0<p<\infty)$ or $\ell^{p-}(0<p \leq \infty)$.

i) The functor BAlg $\rightarrow \mathfrak{A} \mathfrak{b}, \mathfrak{A} \mapsto K H_{*}\left(I_{\ell^{1}(\mathfrak{A})}\right)$ is invariant under Höldercontinuous homotopies and we have $K H_{*}\left(I_{S(\mathfrak{A})}\right)=K H_{*}\left(I_{\ell^{1}(\mathfrak{A})}\right)$ for all $S$ as above.

ii) If $\mathfrak{A}$ is a local Banach algebra and $n \geq 0$, then there is a natural split monomorphism

$$
K_{n}^{\text {top }}(\mathfrak{A})>\quad K H_{n}\left(I_{\ell^{1}(\mathfrak{A})}\right)
$$

iii) If $n \leq 0$, then the comparison map

$$
K_{n}\left(I_{S(\mathfrak{A})}\right) \rightarrow K H_{n}\left(I_{S(\mathfrak{A})}\right)
$$

is an isomorphism for every $\mathfrak{A} \in \mathrm{BAlg}$.

Both these theorems rely on a homotopy invariance theorem (Theorem 7.8) which we think is of independent interest. The theorem says that if $F: \mathbb{C}-\mathrm{Alg} \rightarrow \mathfrak{A b}$ is an $M_{2}$-stable, split exact functor and $S \in\left\{c_{0}, \ell^{p}\right\}$, then the functor

$$
\mathrm{BAlg} \rightarrow \mathfrak{A} \mathfrak{b}, \quad \mathfrak{A} \mapsto F\left(I_{S(\mathfrak{A})}\right)
$$

is homotopy invariant. For $S=c_{0}$ it is continuous homotopy invariant, while for $S=\ell^{p}$ it is invariant under Hölder continuous homotopies, with Hölder exponent depending on $p$. For $F=K H_{*}$ we have $K H_{*}\left(I_{\ell p(\mathfrak{A})}\right)=K H_{*}\left(I_{\ell^{1}(\mathfrak{A})}\right)$, and so it is invariant under arbitrary Hölder continuous homotopies. Furthermore, we have the following general result (see Theorem 8.5) about the comparison map $K \rightarrow K H$. Its proof uses the homotopy invariance theorem mentioned above applied to infinitesimal $K$-theory.

Theorem 1.4 Let $\mathfrak{A}$ be a bornological algebra and let $S$ be $c_{0}, \ell^{p}, \ell^{p+}(0<p<\infty)$ or $\ell^{p-}(0<p \leq \infty)$. Then there are long exact sequences $(n \in \mathbb{Z})$

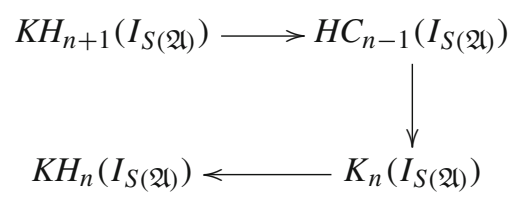

and

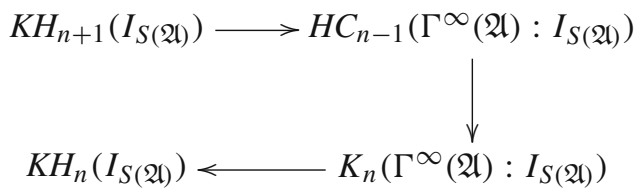


It is shown in the companion paper [6] that $H C_{*}\left(\Gamma^{\infty}(\mathfrak{A}): I_{S(\mathfrak{A})}\right)=0$ when either $S=c_{0}$ and $\mathfrak{A}$ is a $C^{*}$-algebra or $S=\ell^{\infty}$ and $\mathfrak{A}$ is a unital Banach algebra. Therefore, the comparison map $K_{*}\left(I_{S(\mathfrak{A})}\right) \longrightarrow K H_{*}\left(I_{S(\mathfrak{A})}\right)$ is an isomorphism in these cases. In addition, the groups $H C_{n}\left(\Gamma^{\infty}: I_{S}\right)$ are computed in [6] for all $S$, and it is shown that for $S \in\left\{\ell^{p}, \ell^{p \pm}\right\}$ the map $H C_{n}\left(\Gamma^{\infty}: I_{S}\right) \longrightarrow H C_{n}\left(\mathcal{B}: J_{S}\right)$ is an isomorphism for those values of $n$ for which $H C_{n}\left(\mathcal{B}: J_{S}\right)$ was computed by Wodzicki [24]. In summary, the ideals $I_{S} \triangleleft \Gamma^{\infty}$ and $J_{S} \triangleleft \mathcal{B}$ and their corresponding stable algebras have very similar properties in what $K$-theory and cyclic homology are concerned, and the cyclic homology of the former seems to be easier to describe.

We expect that these results will help shed light on the various characters and regulators which take values in the relative $K$-theory and cyclic homology of operator ideals $[9,24]$. This was our original motivation to study the ideals $I_{S}$.

The rest of this paper is organized as follows. In Sect. 2 we establish some notation about sequence spaces, the inverse monoid Emb and the partial isometries $U_{f}$. The algebra $\Gamma^{\infty}(\mathfrak{A})$ and the ideals $I_{S(\mathfrak{A})}$ are introduced in Sect. 3. In this section we also recall the definition of Karoubi's cone $\Gamma(R)$ which is $R$-linearly generated by the $U_{f}$ ( $f \in$ Emb). Proposition 3.5 identifies $I_{S(\mathfrak{A})}$ with a ring formed by certain $\mathbb{N} \times \mathbb{N}$ matrices with coefficients in $\mathfrak{A}$. The two-sided ideals of $\Gamma^{\infty}$ are studied in Sect.4; Theorem 1.1 is contained in Theorem 4.2. We prove in Sect. 5 that if $\mathfrak{A}$ is unital, then $\Gamma^{\infty}(\mathfrak{A})$ is a ring with infinite sums in the sense of Wagoner (Proposition 5.1). In Sect. 6 we show that $I_{S(\mathfrak{A})}$ can be written as a crossed product of $\Gamma=\Gamma(\mathbb{Z})$ and $S(\mathfrak{A})$, by using the conjugation action of Emb in $S(\mathfrak{A})$ via the partial isometries $U_{f}$ (Proposition 6.4). Section 7 deals with the homotopy invariance theorem mentioned above, proved in Theorem 7.8. Applications to $K$-theory are given in Sect. 8; see Theorems 8.1, 8.2 and 8.5.

\section{Preliminaries}

\subsection{Sequence ideals}

Throughout this paper we work in the setting of bornological spaces and bornological algebras; a quick introduction to the subject is given in [12, Chapter 2]. Recall a (complete, convex) bornological vector space over the field $\mathbb{C}$ of complex numbers is a filtering union $\mathbb{V}=\cup_{D} \mathbb{V}_{D}$ of Banach spaces, indexed by the disks of $\mathbb{V}$ such that the inclusions $\mathbb{V}_{D} \subset \mathbb{V}_{D^{\prime}}$ are bounded. A subset of $\mathbb{V}$ is bounded if it is a bounded subset of some $\mathbb{V}_{D}$. A sequence $\mathbb{N} \rightarrow \mathbb{V}$ is bounded if its image is a bounded subset of $\mathbb{V}$. We write $\ell^{\infty}(\mathbb{N}, \mathbb{V})$ or simply $\ell^{\infty}(\mathbb{V})$ for the bornological vector space of bounded sequences where $X \subset \ell^{\infty}(\mathbb{V})$ is bounded if $\bigcup_{x \in X} x(\mathbb{N})$ is. We consider the following subspace

$$
\ell^{\infty}(\mathbb{V}) \supset c_{0}(\mathbb{V})=\left\{\alpha: \lim _{n} \alpha(n)=0\right\}
$$

We equip $c_{0}(\mathbb{V})$ with the bornology induced by that of $\ell^{\infty}(\mathbb{V})$; thus $c_{0}(\mathbb{V}) \subset \ell^{\infty}(\mathbb{V})$ is a closed bornological subspace. We also consider the subspace $(p>0)$ 
$c_{0}(\mathbb{V}) \supset \ell^{p}(\mathbb{V})=\left\{\alpha: \mathbb{N} \rightarrow \mathbb{V}:(\exists \operatorname{a~disk~} D \subset \mathbb{V}) \sum_{n}\|\alpha(n)\|_{D}^{p}<\infty\right\}$

If $p \geq 1$, we equip $\ell^{p}(\mathbb{V})$ with the following bornology: we say that a subset $S \subset \ell^{p}(\mathbb{V})$ is bounded if there is a disk $D$ and a constant $C$ such that $\sum_{n}\|\alpha(n)\|_{D}^{p}<C$ for all $\alpha \in S$. Notice that the inclusion $\ell^{p}(\mathbb{V}) \rightarrow \ell^{\infty}(\mathbb{V})$ is bounded for $p \geq 1$. Recall a bornological algebra is a bornological vector space $\mathfrak{A}$ with an associative bounded multiplication. If $\mathfrak{A}$ is a bornological algebra, then pointwise multiplication makes $\ell^{\infty}(\mathfrak{A})$ into a bornological algebra, $c_{0}(\mathfrak{A}) \triangleleft \ell^{\infty}(\mathfrak{A})$ is a closed bornological ideal, and $\ell^{p}(\mathfrak{A}) \triangleleft \ell^{\infty}(\mathfrak{A})$ is an algebraic ideal for all $p>0$.

Notation 2.1 When $\mathfrak{A}$ is $\mathbb{C}$, we shall omit it from our notation. Thus we shall write $\ell^{\infty}, \ell^{p}, c_{0}$, etc, for $\ell^{\infty}(\mathbb{C}), \ell^{p}(\mathbb{C}), c_{0}(\mathbb{C})$, etc.

The space $\mathcal{B}\left(\ell^{2}(\mathbb{V})\right)$ of bounded operators $\ell^{2}(\mathbb{V}) \rightarrow \ell^{2}(\mathbb{V})$ on a bornological vector space $\mathbb{V}$ is a bornological algebra with the uniform bornology [12, Def. 2.4]. If $\mathfrak{A}$ is a bornological algebra, then

$$
\operatorname{diag}: \ell^{\infty}(\mathfrak{A}) \rightarrow \mathcal{B}\left(\ell^{2}(\mathfrak{A})\right), \quad \operatorname{diag}(\alpha)(\xi)=\left(\alpha(n) \xi_{n}\right)_{n \geq 1}
$$

is a bounded representation. It is faithful if and only if the left annihilator of $\mathfrak{A}$ is trivial:

$$
\operatorname{ann}(\mathfrak{A})=\{a \in \mathfrak{A}: a \cdot b=0 \quad(\forall b \in \mathfrak{A})\}=0,
$$

This happens, for instance, when $\mathfrak{A}$ is unital.

\subsection{The monoid Emb}

We begin by recalling some definitions from [14]. We denote by Emb the set of injective functions

$$
\mathrm{Emb}=\{f: A \longmapsto \mathbb{N}: A \subset \mathbb{N}\}
$$

Note that Emb is a monoid for the composition law:

$$
f g: \operatorname{dom}(g) \cap g^{-1}(\operatorname{dom}(f)) \rightarrow \mathbb{N},(f g)(n)=f(g(n)) .
$$

In (2.3) and elsewhere, we shall omit the composition sign o, except when strictly necessary to avoid confusion. The monoid Emb is pointed, i.e. it has a zero element, namely, the empty function $\emptyset \rightarrow \mathbb{N}$. The antipode map ${ }^{\dagger}:$ Emb $\rightarrow$ Emb is defined by

$$
\operatorname{dom}\left(f^{\dagger}\right)=\operatorname{ran}(f), f^{\dagger}(n)=f^{-1}(n)
$$

If $A \subset \mathbb{N}$, we write $P_{A}$ for the inclusion $A \hookrightarrow \mathbb{N}$. It is easily checked that

$$
f^{\dagger} f=P_{\operatorname{dom} f}, f f^{\dagger}=P_{\operatorname{ran} f},
$$


for any $f \in$ Emb. Observe that $f^{\dagger}$ is characterized as the unique element of Emb which satisfies simultaneously

$$
f f^{\dagger} f=f \quad \text { and } \quad f^{\dagger} f f^{\dagger}=f^{\dagger} .
$$

Thus the monoid Emb together with its antipode is a pointed inverse monoid that is, a pointed inverse semigroup with identity element. Note that Emb is the object usually denoted $\mathcal{I}(\mathbb{N})$ in the literature on semigroups (see [15, Def. 4.2], for instance).

If $\mathbb{V}$ is a bornological vector space, the monoid Emb acts on $\ell^{\infty}(\mathbb{V})$ via:

$$
f_{*}(\alpha)_{n}= \begin{cases}\alpha\left(f^{\dagger}(n)\right) & \text { if } n \in \operatorname{ran}(f) \\ 0 & \text { otherwise }\end{cases}
$$

The subspaces $c_{0}(\mathbb{V})$ and $\ell^{p}(\mathbb{V})$ defined in 2.1 are symmetric, i.e. they are invariant under the action of Emb. Indeed, this follows from the fact that $c_{0}$ and $\ell^{p}$ are symmetric, and that if $D$ is a bounded disk and the image of $\alpha$ is contained in $\mathbb{V}_{D}$, then the following sequences of real numbers are identical

$$
\left\|f_{*}(\alpha)\right\|_{D}=f_{*}\left(\|\alpha\|_{D}\right) .
$$

More generally, if $S \subset \ell^{\infty}$ is any symmetric subspace, then

$$
S(\mathbb{V}):=\left\{\alpha \in \ell^{\infty}(\mathbb{V}):(\exists D) \alpha(\mathbb{N}) \subset \mathbb{V}_{D} \text { and }\|\alpha\|_{D} \in S\right\}
$$

is symmetric. We denote by $U$ the representation of Emb by partial isometries on $\ell^{2}(\mathbb{V})$ :

$$
U_{f}(\xi)_{m}=\left\{\begin{array}{ll}
\xi_{n} & \text { if } f(n)=m \\
0 & \text { if } m \notin \operatorname{ran}(f)
\end{array} \quad\left(\xi \in \ell^{2}(\mathbb{V})\right)\right.
$$

Straightforward computations show that

$$
U_{f g}=U_{f} U_{g}
$$

Observe that $U_{f}$ is a partial isometry whose initial and final space are, respectively, the closed subspaces

$$
\operatorname{span}\{v: \operatorname{supp}(v) \subset \operatorname{dom}(f)\} \text { and } \operatorname{span}\{v: \operatorname{supp}(v) \subset \operatorname{ran}(f)\} .
$$

This follows from (2.4), (2.7), and from the fact that if $A \subset \mathbb{N}$, then

$$
U_{P_{A}}(v)_{n}= \begin{cases}v_{n} & \text { if } n \in A \\ 0 & \text { otherwise }\end{cases}
$$


Remark 2.2 We will often work with sequences indexed by infinite countable sets other than $\mathbb{N}$. A bijection $u: \mathbb{N} \rightarrow X$ gives rise to a bounded isomorphism $\alpha \mapsto \alpha u$ between the bornological vector space $\ell^{\infty}(X, \mathbb{V})$ of bounded maps from $X$ to the bornological space $\mathbb{V}$ and the space $\ell^{\infty}(\mathbb{V})=\ell^{\infty}(\mathbb{N}, \mathbb{V})$. If $S \subset \ell^{\infty}$ is a symmetric subspace, we define $S(X, \mathbb{V})=\left\{s u^{-1}: s \in S(\mathbb{V})\right\}$. Because $S$ is symmetric by assumption, this definition does not depend on the choice of $u$. If $A \in M_{\mathbb{N} \times \mathbb{N}}(\mathbb{V})$, we will write $A \in S(\mathbb{N} \times \mathbb{N}, \mathbb{V})$ to indicate that $\left\{A_{i j}: i, j \in \mathbb{N}\right\} \in S(\mathbb{N} \times \mathbb{N}, \mathbb{V})$.

Notation 2.3 Let $S \subset \ell^{\infty}$ be a symmetric subspace, $X$ an infinite countable set and $\mathbb{V}$ a bornological vector space. We use the following abbreviated notation: $S=S(\mathbb{N}, \mathbb{C})$, $S(X)=S(X, \mathbb{C})$ and $S(\mathbb{V})=S(\mathbb{N}, \mathbb{V})$.

\section{The algebras $\Gamma^{\infty}(\mathfrak{A})$ and $\Gamma(R)$}

Throughout this section, $\mathfrak{A}$ will be a fixed bornological algebra, which, except in Definition 3.7, will be assumed unital. It follows straightforwardly from equations (2.2), (2.5), and (2.6) that

$$
\operatorname{diag}\left(f_{*}(\alpha)\right) U_{f}=U_{f} \operatorname{diag}(\alpha) \text { and } U_{f} \operatorname{diag}(\alpha) U_{f^{\dagger}}=\operatorname{diag}\left(f_{*}(\alpha)\right)
$$

where $\alpha \in \ell^{\infty}(\mathfrak{A})$ and $f \in$ Emb. Set

$$
\Gamma^{\infty}(\mathfrak{A})=\operatorname{span}\left\{\operatorname{diag}(\alpha) U_{f}: \alpha \in \ell^{\infty}(\mathfrak{A}), \quad f \in \mathrm{Emb}\right\} .
$$

Notice that, by Eqs. (2.7) and (3.1), $\Gamma^{\infty}(\mathfrak{A})$ is a subalgebra of the algebra $\mathcal{B}\left(\ell^{2}(\mathfrak{A})\right)$. For each symmetric ideal $S \triangleleft \ell^{\infty}$, we write $I_{S(\mathfrak{A})}$ for the ideal of $\Gamma^{\infty}(\mathfrak{A})$ generated by $\operatorname{diag}(S(\mathfrak{A}))$. Because $S$ is invariant under the action of Emb, then by equations (3.1) we have

$$
I_{S(\mathfrak{A})}=\operatorname{span}\left\{\operatorname{diag}(\alpha) U_{f}: \alpha \in S(\mathfrak{A}), f \in \mathrm{Emb}\right\} .
$$

Note that $\Gamma^{\infty}(\mathfrak{A})=I_{\ell}(\mathfrak{A})$. If $X$ is any infinite countable set, we may also consider the subalgebra $\Gamma^{\infty}(X, \mathfrak{A}) \subset \mathcal{B}\left(\ell^{2}(X, \mathfrak{A})\right)$ spanned by $\operatorname{diag}\left(\ell^{\infty}(X, \mathfrak{A})\right)$ and $U_{\operatorname{Emb}(X)}$. Thus $\Gamma^{\infty}(\mathfrak{A})=\Gamma^{\infty}(\mathbb{N}, \mathfrak{A})$. In keeping with our notational conventions 2.1 and 2.3, we write $\Gamma^{\infty}=\Gamma^{\infty}(\mathbb{C})$ and $\Gamma^{\infty}(X)=\Gamma^{\infty}(X, \mathbb{C})$.

Notation 3.1 Since $\mathfrak{A}$ is assumed to be unital, every sequence $a=\left\{a_{n}\right\}$ in $\ell^{2}(\mathfrak{A})$ can be written uniquely as $a=\sum_{n} a_{n} e_{n}$, where $e_{n} \in \ell^{2}(\mathfrak{A})$ is defined by $\left(e_{n}\right)_{i}=\delta_{n, i}$. Notice that the elements of $\Gamma^{\infty}(\mathfrak{A})$ are $\mathfrak{A}$-linear operators on the right $\mathfrak{A}$-module $\ell^{2}(\mathfrak{A})$. As usual, we identify an $\mathfrak{A}$-linear operator $A \in \mathcal{B}\left(\ell^{2}(\mathfrak{A})\right)$ with the infinite matrix $\left(A_{i j}\right)_{i, j \in \mathbb{N}}$ with entries in $\mathfrak{A}$ defined by

$$
A e_{n}=\sum_{k} A_{k n} e_{k}
$$


We denote by $E_{i j}$ the matrix $\left(E_{i j}\right)_{k l}=\delta_{i k} \delta_{j l}$. Given a matrix $A=\left(A_{i j}\right)_{i, j \in \mathbb{N}}$ with entries in $\mathfrak{A}$, and $i, j \in \mathbb{N}$, we set:

$$
\begin{aligned}
J_{i}(A) & =\left\{j: A_{i j} \neq 0\right\}, \quad I_{j}(A)=\left\{i: A_{i j} \neq 0\right\}, \\
r_{i}(A) & =\# J_{i}(A), \quad c_{j}(A):=\# I_{j}(A), \\
r(A) & :=\max _{i} r_{i}(A), \quad c(A):=\max _{i} c_{i}(A), \\
N(A) & :=\max \{r(A), c(A)\},
\end{aligned}
$$

where $r_{i}(A), c_{j}(A), N(A) \in \mathbb{N} \cup\{\infty\}$. If $R$ is a ring, we write $\Gamma(R)$ for Karoubi's cone

$$
\Gamma(R)=\left\{A \in R^{\mathbb{N} \times \mathbb{N}}: N(A)<\infty \text { and }\left\{A_{i, j}: i, j \in \mathbb{N}\right\} \text { is finite }\right\}
$$

It was shown in $[8$, Lemma 4.7.1] that $\Gamma(R)$ is isomorphic to $R \otimes \Gamma(\mathbb{Z})$, for any ring $R$. We shall write

$$
\Gamma=\Gamma(\mathbb{Z})
$$

Observe that definition (3.4) extends to matrices indexed by any countable infinite set $X$; if $f: \mathbb{N} \rightarrow X$ is a bijection, $\Gamma(X, R) \subset R^{X \times X}$ is the image of $\Gamma(R)$ under the map $A \mapsto U_{f} A U_{f^{-1}}$. Thus $\Gamma(R)=\Gamma(\mathbb{N}, R)$; we shall write $\Gamma(X)=\Gamma(X, \mathbb{Z})$.

The following lemmas will be useful in obtaining characterizations of $\Gamma^{\infty}(\mathfrak{A}), I_{S(\mathfrak{A})}$ and $\Gamma(R)$ as rings of matrices acting on $\ell^{2}(\mathfrak{A})$ and $R^{(\mathbb{N})}$, respectively. If $A \in R^{\mathbb{N} \times \mathbb{N}}$ is such that $N(A)<\infty$, we write $\Gamma(R) A \Gamma(R)$ to denote the set

$$
\Gamma(R) A \Gamma(R):=\left\{\sum_{j=1}^{n} P_{j} A Q_{j}: P_{j}, Q_{j} \in \Gamma(R) \text { for all } j=1, \ldots, n \text { and } n \in \mathbb{N}\right\} .
$$

Lemma 3.2 Let $R$ be a unital ring, $A=\left(A_{i j}\right)_{i, j \in \mathbb{N}} \in R^{\mathbb{N} \times \mathbb{N}}$ a matrix such that $N(A)<\infty$ and $r(A)>1$. Then

(1) $A=A_{1}+A_{2}+\cdots+A_{k}$, where $A_{i} \in \Gamma(R) A \Gamma(R), r\left(A_{i}\right)<r(A)$ and $c\left(A_{i}\right) \leq$ $c(A)$ for all $i=1, \ldots, k$.

(2) If in addition $R$ is a unital bornological algebra and $S \triangleleft \ell^{\infty}$ is a symmetric ideal such that $A \in S(\mathbb{N} \times \mathbb{N}, R)$, then $A_{l} \in S(\mathbb{N} \times \mathbb{N}, R)$, for all $l=1, \ldots, k$.

Proof (1) We first establish some notation and make some reductions. Let

$$
\begin{aligned}
& r=r(A) \\
& I=\{i \in \mathbb{N}: \text { the } i \text { th row of } A \text { has } r \text { nonzero entries }\} .
\end{aligned}
$$

For $i \in I$, let

$$
h_{i}(1)<h_{i}(2)<\cdots<h_{i}(r)
$$


be the columns where the nonzero entries of row $i$ occur. Let $A_{r}$ denote the matrix obtained from $A$ upon multiplying by zero those rows that have less than $r$ nonzero entries. Then $A_{r} \in \Gamma(R) A \Gamma(R)$, and

$$
r\left(A_{r}\right)=r, r\left(A-A_{r}\right)<r, c\left(A_{r}\right) \leq c(A), \text { and } c\left(A-A_{r}\right) \leq c(A) .
$$

Thus it suffices to prove (1) for $A_{r}$. Hence we may assume that $A=A_{r}$, that is, that all nonzero rows of $A$ have exactly $r$ nonzero entries. Furthermore, since there are at most $c(A)$ nonzero entries in each column of $A$, the set $I$ can be written as a disjoint union $I=I_{1} \sqcup I_{2} \sqcup \cdots \sqcup I_{s}$ with $s \leq c(A)$ and such that each $I_{t}(1 \leq t \leq s)$ satisfies the following property:

$$
i \neq j \in I_{t} \Rightarrow h_{i}(1) \neq h_{j}(1)
$$

Proceeding as above we see that we may assume that $s=1$. Notice that if $A^{\prime}$ is obtained from $A$ by permuting its rows, then $A^{\prime}=U_{f} A$ for some bijection $f: \mathbb{N} \rightarrow \mathbb{N}$. Therefore, $\Gamma(R) A \Gamma(R)=\Gamma(R) A^{\prime} \Gamma(R), r\left(A^{\prime}\right)=r(A)$, and $c\left(A^{\prime}\right)=c(A)$, so we may assume that $A=A^{\prime}$. Thus we will assume that the rows of $A$ are ordered so that if $i, j \in I$, then $h_{i}(1)<h_{j}(1)$ if and only if $i<j$.

Thus, it only remains to show (1) for matrices $A$ such that for $I$ and $h_{i}$ as above:

(a) All nonzero rows of A have exactly $\mathrm{r}$ nonzero entries.

(b) $i<j \Longleftrightarrow h_{i}(1)<h_{j}$ (1) for all $i, j \in I$.

We shall proceed by induction on

$$
M_{A}=\max _{j \in I} \#\left\{i \in I: A_{i h_{j}(1)} \neq 0\right\} .
$$

Notice that the right-hand side of the equation above is bounded by $c(A)$, so $M_{A} \in \mathbb{N}$. First assume that $M_{A}=1$. Then for all $i, j \in I$ we have that $A_{i h_{j}(1)} \neq 0$ if and only if $i=j$. Set

$$
A_{1}=\sum_{i \in I} A_{i h_{i}(1)} E_{i h_{i}(1)}=\left(\sum_{i \in I} E_{i i}\right) A\left(\sum_{j \in I} E_{h_{j}(1) h_{j}(1)}\right) \in \Gamma(R) A \Gamma(R) .
$$

Then

$$
r\left(A_{1}\right)<r, r\left(A-A_{1}\right)<r, c\left(A_{1}\right) \leq c(A), \text { and } c\left(A-A_{1}\right) \leq c(A),
$$

so the statement in (1) holds for $A$. Assume now that $M_{A}>1$ and that (1) holds for matrices $B$ satisfying 3.5 and 3.6, and such that $M_{B}<M_{A}$. Let

$$
i_{1}:=\min I, K_{1}:=\left\{j \in I: A_{i_{1} h_{j}(1) \neq 0}\right\}
$$


For $n \geq 1$ such that $\bigcup_{j=1}^{n-1} K_{j} \neq I$, let

$$
i_{n}:=\min I \backslash \bigcup_{j=1}^{n-1} K_{j} \text {, and } K_{n}:=\left\{j \in I \backslash \bigcup_{l=1}^{n-1} K_{l}: A_{i_{n} h_{j}(1)} \neq 0\right\} .
$$

Let

$$
\mathcal{J}=\left\{\begin{array}{l}
\{1,2, \ldots, n\}, \text { if } \bigcup_{j=1}^{n} K_{j}=I \\
\mathbb{N}, \text { otherwise }
\end{array}\right.
$$

We claim that

$$
\text { a) } i_{n}>i_{n-1} \forall n \in \mathcal{J} \quad \text { and } \quad \text { b) } I=\bigcup_{j \in \mathcal{J}} K_{j} \text {. }
$$

In fact a) follows from the inequality

$$
i_{n}=\min I \backslash \bigcup_{1}^{n-1} K_{j} \geq \min I \backslash \bigcup_{1}^{n-2} K_{j}=i_{n-1}
$$

and the fact that $i_{n} \neq i_{n-1}$ because $i_{n} \notin K_{n-1}$ and $i_{n-1} \in K_{n-1}$. It is clear that b) holds when $\mathcal{J}$ is finite. Assume now that $\mathcal{J}$ infinite. If $k \in I$, then either $k \in\left\{i_{n}: n \in\right.$ $\mathcal{J}\} \subset \bigcup K_{j}$ or, by a), there exists $n \in \mathcal{J}$ such that

$$
k<i_{n}=\min I \backslash \bigcup_{1}^{n-1} K_{j}
$$

This implies that $k \in \bigcup_{1}^{n-1} K_{j}$. Thus b) holds also when $\mathcal{J}$ is infinite, and both claims are proven. Now set

$$
B:=\sum_{n \in \mathcal{J}, j \in \mathbb{N}} A_{i_{n} j} E_{i_{n} j}=\left(\sum_{n \in \mathcal{J}} E_{i_{n} i_{n}}\right) A \in \Gamma(R) A \Gamma(R) .
$$

Notice that $B$ is obtained from $A$ by multiplying by zero the $i^{\text {th }}$ row whenever $i \notin$ $\left\{i_{n}: n \in \mathcal{J}\right\}$. Therefore $B$ satisfies 3.5 and 3.6, $r(B)=r$, and $c(B) \leq c(A)$. We next show that $M_{B}=1$. We begin by noting that $B_{i_{m} i_{n}(1)} \neq 0$ implies that $A_{i_{m} i_{n}(1)} \neq 0$. Then $i_{n}(1) \geq i_{m}(1)$, which implies by 3.6 that $i_{n} \geq i_{m}$, which in turn implies, by part a) of Eq. (3.7), that $n \geq m$. Now, if $n>m$ we would have

$$
i_{n} \notin \bigcup_{1}^{n-1} K_{j} \supseteq \bigcup_{1}^{m} K_{j} .
$$


Then $i_{n} \notin K_{m}$ and $i_{n} \notin \bigcup_{1}^{m-1} K_{j}$, which implies that $A_{i_{m} i_{n}(1)}=0$, a contradiction. Thus $n=m$ and $M_{B}=1$, as claimed. Set $C=A-B$; we have $r(C)=r$ and $c(C) \leq c(A)$. Notice that $C$ is obtained from $A$ upon multiplying by zero the $i_{n}^{\text {th }}$ row for all $n \in \mathcal{J}$. Besides, the $i^{t h}$ row of $C$ is nonzero if and only if $i \in I_{C}:=I \backslash\left\{i_{n}: n \in \mathcal{J}\right\}$, and in that case it is equal to the $i^{\text {th }}$ row of $A$. Therefore, $C$ satisfies 3.5 and 3.6. We next prove that $M_{C}<M_{A}$, which will conclude the proof of part (1). If $i, j \in I_{C}$, then $A_{i h_{j}(1)}=0$ implies that $C_{i h_{j}(1)}=0$. On the other hand, by part b) of Eq. (3.7), we can choose $n \in \mathcal{J}$ such that $j \in K_{n}$. Then $A_{i_{n} h_{j}(1)} \neq 0$, whereas $C_{i_{n} h_{j}(1)}=0$. It follows that $M_{C} \leq M_{A}-1$. This concludes the proof of part (1). Part (2) holds because for $l=1, \ldots, k,\left\{\left(A_{l}\right)_{i j}\right\}$ is obtained upon multiplication of $\left\{A_{i j}\right\}$ by bounded sequences and by permutations of terms.

Lemma 3.3 Let $A=\left(A_{i j}\right)_{i, j \in \mathbb{N}}$ be a matrix with entries in a unital ring $R$ such that $N(A)<\infty$. Then

(1) $A=A_{1}+A_{2}+\cdots+A_{k}$, where $A_{i} \in \Gamma(R) A \Gamma(R)$, and $N\left(A_{i}\right) \leq 1$, for all $i=1, \ldots, k$.

(2) If in addition $R$ is a bornological algebra and $S \triangleleft \ell^{\infty}$ is a symmetric ideal such that $A \in S(\mathbb{N} \times \mathbb{N}, R)$, then $A_{l} \in S(\mathbb{N} \times \mathbb{N}, R)$, for all $l=1, \ldots, k$.

Proof Use Lemma 3.2 and proceed by induction on $r(A)$ to write

$$
A=\sum_{1}^{k} B_{i}, \text { where } r\left(B_{i}\right)=1, c\left(B_{i}\right) \leq c(A) \text {, and } B_{i} \in \Gamma(R) A \Gamma(R) \text {. }
$$

Next apply the same procedure to each transpose matrix $B_{i}^{t}$ to get the decomposition in (1). The second statement follows from the second part of Lemma 3.2.

Proposition 3.4 Let $A=\left(A_{i j}\right)_{i, j \in \mathbb{N}}$ be a matrix with entries in a ring $R$. Then $N(A) \leq 1$ if and only if $A=\operatorname{diag}(\alpha) U_{f}$, where $f \in \operatorname{Emb}$ and $\alpha \in R^{\mathbb{N}}$ are defined as follows:

$$
f(j)=i \Longleftrightarrow A_{i j} \neq 0 \quad \alpha(i)= \begin{cases}A_{i j}, & \text { if } i=f(j) \\ 0, & \text { otherwise. }\end{cases}
$$

Proof For $f$ and $\alpha$ as in the proposition, the $n$th column of $A$ is

$$
\begin{aligned}
\left(\operatorname{diag}(\alpha) U_{f}\right)\left(e_{n}\right) & = \begin{cases}\alpha(n) e_{f(n)}, & \text { if } n \in \operatorname{dom}(f) \\
0, & \text { otherwise. }\end{cases} \\
& = \begin{cases}A_{f(n) n} e_{f(n)}, & \text { if } n \in \operatorname{dom}(f) \\
0, & \text { otherwise. }\end{cases}
\end{aligned}
$$


Proposition 3.5 Let $\mathfrak{A}$ be a unital bornological algebra, $S \triangleleft \ell^{\infty}$ a symmetric ideal, and $I_{S(\mathfrak{A})} \triangleleft \Gamma^{\infty}(\mathfrak{A})$ the ideal defined in Eq. (3.3). Then

$$
I_{S(\mathfrak{A})}=\left\{A=\left(A_{i j}\right)_{i, j \in \mathbb{N}}: A \in S(\mathbb{N} \times \mathbb{N}, \mathfrak{A}) \text { and } N(A)<\infty\right\} .
$$

Proof Let $D_{S}$ denote the set on the right hand side of equation (3.8). By Lemma 3.3 and Proposition 3.4, a matrix $A$ belongs to $D_{S}$ if and only if $A=\sum A_{k}$, with $A_{k}=$ $\operatorname{diag}\left(\alpha_{k}\right) U_{f_{k}} \in D_{S}$. Further, we may choose $\alpha_{k}$ and $f_{k} \operatorname{such}$ that $\operatorname{supp}\left(\alpha_{k}\right)=\operatorname{ran}\left(f_{k}\right)$. Under these conditions, $A_{k} \in D_{S}$ if and only if $\alpha_{k} \in S$. This shows that $A \in D_{S}$ if and only $A \in I_{S}$.

Corollary 3.6 Let $\mathfrak{A}$ be a unital bornological algebra. Then Karoubi's cone $\Gamma(\mathfrak{A})$ is a subalgebra of $\Gamma^{\infty}(\mathfrak{A})$.

Definition 3.7 If $\mathfrak{A}$ is a not necessarily unital bornological algebra, and $S \triangleleft \ell^{\infty}$ is a symmetric ideal, $I_{S(\mathfrak{A})}$ is defined by (3.8).

Example 3.8 Let

$$
c_{f}=\left\{\alpha \in \ell^{\infty}: \operatorname{supp}(\alpha) \text { is finite }\right\}
$$

Then

$$
I_{c_{f}(\mathfrak{A})}=M_{\infty}(\mathfrak{A})=\left\{A: \exists n \in \mathbb{N} \text { such that } A_{i j}=0 \text { if either } i>n \text { or } j>n\right\} .
$$

We shall write $M_{\infty}=M_{\infty} \mathbb{Z}$.

Remark 3.9 Let $\mathfrak{A}$ be a unital bornological algebra, $I \triangleleft \Gamma^{\infty}(\mathfrak{A})$ a two-sided ideal and $T \in I$. Then by Lemma 3.3 and Remark 3.4, we can write

$$
T=\sum_{i=1}^{n} \operatorname{diag}\left(\alpha_{i}\right) U_{f_{i}} \text { with } \operatorname{diag}\left(\alpha_{i}\right) U_{f_{i}} \in I,
$$

where $f_{i} \in$ Emb and $\alpha_{i} \in \ell^{\infty}(\mathfrak{A})$. Similarly, if $R$ is a unital ring and $T \in I \triangleleft \Gamma(R)$, then we can also write $T$ as in (3.9) but now with $\alpha_{i}$ such that the $\operatorname{set}\left\{\alpha_{i}(n): n \in\right.$ $\mathbb{N}\} \subset R$ is finite.

\section{The two-sided ideals of $\Gamma^{\infty}$ and those of $\mathcal{B}\left(\ell^{2}(\mathbb{N})\right)$}

Calkin's theorem [2, Theorem 1.6]), as restated by Garling [16, Theorem 1], establishes a bijective correspondence between the set of proper two-sided ideals of $\mathcal{B}=\mathcal{B}\left(\ell^{2}\right)$ and the set of proper symmetric ideals of $\ell^{\infty}$. Calkin defined this correspondence in terms of the sequence of singular values of a compact operator. It can also be described as follows: an ideal $J \triangleleft \mathcal{B}$ is mapped to the symmetric ideal

$$
S(J)=\left\{\alpha \in \ell^{\infty}: \operatorname{diag}(\alpha) \in J\right\} .
$$


The inverse correspondence maps a symmetric ideal $S$ in $\ell^{\infty}$ to the two-sided ideal

$$
\mathcal{B} \triangleright J_{S}=\langle\operatorname{diag}(\alpha): \alpha \in S\rangle
$$

We refer the reader to [20, Theorem 2.5] for further details. Recall that, by another result of Calkin [2, Theorem 1.4], the Calkin algebra $\mathcal{B} / \mathcal{K}$ is simple. On the other hand, it is easily checked that $c_{0} \triangleleft \ell^{\infty}$ is maximal among proper symmetric ideals. Thus, by mapping $\ell^{\infty}$ to $\mathcal{B}$ we extend the correspondence above to a bijection between the family of symmetric ideals of $\ell^{\infty}$ and that of two-sided ideals of $\mathcal{B}$. In Theorem 4.2 below we show that Calkin's correspondence carries over to ideals in $\Gamma^{\infty}$. We will make use of the following lemma.

Lemma 4.1 Let $\alpha \in \ell^{\infty}, f \in \mathrm{Emb}$ and let $I \triangleleft \Gamma^{\infty}$ a two-sided ideal. Consider the operator

$$
T=\operatorname{diag}(\alpha) U_{f}
$$

Then

$$
T \in I \Longleftrightarrow|T| \in I \text {. }
$$

Proof We have

$$
T^{*} T=U_{f}^{*} \operatorname{diag}\left(|\alpha|^{2}\right) U_{f}=\operatorname{diag}\left(f_{*}^{\dagger}\left(|\alpha|^{2}\right)\right)=\operatorname{diag}\left(\left|f_{*}^{\dagger}(\alpha)\right|^{2}\right) .
$$

Therefore, $|T|=\operatorname{diag}\left(\left|f_{*}^{\dagger}(\alpha)\right|\right)$, and the polar decomposition of $T$ is $T=V|T|$, where

$$
V=\operatorname{diag}\left(v_{\alpha}\right) U_{f}
$$

for

$$
v_{\alpha}(n)= \begin{cases}0, & \text { if } \alpha(n)=0 \\ \frac{\alpha(n)}{|\alpha(n)|}, & \text { otherwise. }\end{cases}
$$

It is now clear that $V \in \Gamma^{\infty}$. Thus $T \in I$ if and only if $|T| \in I$, since $\Gamma^{\infty}$ is a *-algebra and $|T|=V^{*} T$.

Theorem 4.2 i) The map $S \mapsto I_{S}$ is a bijection between the set of symmetric ideals of $\ell^{\infty}$ and the set of two-sided ideals of $\Gamma^{\infty}$. Its inverse maps an ideal $I \triangleleft \Gamma^{\infty}$ to the symmetric ideal $S(I)$ defined as in (4.1).

ii) The map $J \mapsto J \cap \Gamma^{\infty}$ is a bijection between the sets of two-sided ideals of $\mathcal{B}$ and those of $\Gamma^{\infty}$. Its inverse maps an ideal $I \triangleleft \Gamma^{\infty}$ to the two-sided ideal of $\mathcal{B}$ it generates.

iii) If $S \triangleleft \ell^{\infty}$ is a symmetric ideal, then $J_{S} \cap \Gamma^{\infty}=I_{S}$. 
Proof Let $I \triangleleft \Gamma^{\infty}$; write $S=S(I)$. It is clear that $I_{S} \subseteq I$. On the other hand, if $T=\operatorname{diag}(\alpha) U_{f} \in I$, for some $\alpha \in \ell^{\infty}$ and $f \in$ Emb, then, by Lemma 4.1,

$$
\operatorname{diag}\left(f_{*}^{\dagger}(|\alpha|)\right)=|T| \in I_{S}
$$

Hence $T \in I_{S}$, again by Lemma 4.1. In view of Remark 3.9, this implies that $I=I_{S}$. We have shown that $I_{S(I)}=I$. Let now $S \triangleleft \ell^{\infty}$ be a symmetric ideal. Then

$$
S \subset S\left(I_{S}\right) \subset S\left(J_{S}\right) \subset S
$$

the last inclusion being due to Calkin's theorem. It follows that $S=S\left(I_{S}\right)$, completing the proof of part i). Next, since the ideal $\left\langle I_{S}\right\rangle \triangleleft \mathcal{B}\left(\ell^{2}\right)$ generated by $I_{S}$ is also generated by $\operatorname{diag}(S)$ we have $\left\langle I_{S}\right\rangle=J_{S}$, by Calkin's theorem. Now, again by Calkin's theorem,

$$
S \subset S\left(J_{S} \cap \Gamma^{\infty}\right) \subset S\left(J_{S}\right)=S .
$$

Thus $J_{S} \cap \Gamma^{\infty}=I_{S}$, by part i). We have proven part iii) and also shown that $\left\langle I_{S}\right\rangle \cap \Gamma^{\infty}=$ $I_{S}$. Moreover, by parts i) and iii) we have

$$
\operatorname{diag}\left(\ell^{\infty}\right) \cap J_{S}=\operatorname{diag}\left(\ell^{\infty}\right) \cap J_{S} \cap \Gamma^{\infty}=\operatorname{diag}\left(\ell^{\infty}\right) \cap I_{S}=\operatorname{diag}(S) .
$$

It follows that $\left\langle J_{S} \cap \Gamma^{\infty}\right\rangle=J_{S}$, which ends the proof.

It follows from Proposition 3.5, Example 3.8 and Theorem 4.2 that

$$
I \cap \Gamma(\mathbb{C})=M_{\infty}(\mathbb{C})
$$

for every proper ideal $I \triangleleft \Gamma^{\infty}$. The next proposition shows that in fact $M_{\infty}(\mathbb{C})$ is the only proper ideal of $\Gamma(\mathbb{C})$.

Proposition 4.3 Let $k$ be a field. Then $M_{\infty}(k)$ is the only proper two-sided ideal of $\Gamma(k)$.

Proof It is well known and easy to check that $M_{\infty}(R) \triangleleft \Gamma(R)$ for any ring $R$. Let $I \neq 0$ be a two-sided ideal of $\Gamma(k)$, and let $A \neq 0, A \in I$. If $i_{0}$ and $j_{0}$ are such that $A_{i_{0} j_{0}} \neq 0$, then

$$
E_{i j}=\left(A_{i_{0} j_{0}}\right)^{-1} E_{i i_{0}} A E_{j_{0} j} \in I \quad \forall i, j
$$

This shows that $M_{\infty}(k) \subseteq I$. Assume that the inclusion is strict. Let $A \in I \backslash M_{\infty}(k)$. By Remark (3.9), we may assume that $A=\operatorname{diag}(\alpha) U_{f}$ for $f \in \operatorname{Emb}$ and $\alpha \in k^{\mathbb{N}}$, where $\operatorname{Im}(\alpha)=\{\alpha(n): n \in \mathbb{N}\}$ is finite and $\operatorname{supp}(\alpha)=\operatorname{dom} f \subset \mathbb{N}$ is infinite. Because $k$ is a field, we can multiply $A$ on the left by a diagonal matrix in $\Gamma(k)$ to conclude that $U_{f} \in I$. But since $\operatorname{ran}(f)$ is infinite, there are bijections $g: \mathbb{N} \rightarrow \operatorname{dom}(f)$ and $h: \operatorname{ran}(f) \rightarrow \mathbb{N}$ such that $h f g=1$. Hence $I$ must contain $1=U_{h} U_{f} U_{g}$. 


\section{$5 \Gamma^{\infty}$ as an infinite sum ring}

We begin this section by recalling some definitions from [22] and [8]. A sum ring $\left(R, x_{0}, x_{1}, y_{0}, y_{1}\right)$ consists of a unital ring $R$ and elements $x_{0}, x_{1}, y_{0}$, and $y_{1} \in R$ satisfying:

$$
\begin{aligned}
& y_{0} x_{0}=y_{1} x_{1}=1 \\
& x_{0} y_{0}+x_{1} y_{1}=1 .
\end{aligned}
$$

If $R$ is a sum ring, the map

$$
\oplus: R \times R \longrightarrow R \text {, defined by } r \oplus s=x_{0} r y_{0}+x_{1} s y_{1},
$$

is a unital ring homomorphism. An infinite sum ring consists of a sum ring $R$ equipped with a unital ring homomorphism

$$
\Phi: R \longrightarrow R \text { such that } r \oplus \Phi(r)=\Phi(r)
$$

The notion of infinite sum ring was introduced by Wagoner [22]. He showed that if $R$ is unital, then the following is an infinite sum ring:

$$
\Gamma^{W}(R):=\left\{A \in R^{\mathbb{N} \times \mathbb{N}}: A \cdot M_{\infty} R \subset M_{\infty} R \supset M_{\infty} R \cdot A\right\}
$$

We may regard $\Gamma^{W}(R)$ as a multiplier algebra of $M_{\infty} R$. One checks that a matrix $A \in \Gamma^{W}(R)$ if and only if every row and every column of $A$ has finite support. Let

$$
f_{i}: \mathbb{N} \rightarrow \mathbb{N}, f_{i}(n)=2 n-i \quad(i=0,1)
$$

The elements $x_{i}=U_{f_{i}^{\dagger}}, y_{i}=U_{f_{i}}$ satisfy conditions (5.1). The homomorphism $\Phi$ is defined by

$$
\Phi(A)=\sum_{k=0}^{\infty} x_{1}^{k} x_{0} A y_{0} y_{1}^{k}=\sum_{k, i, j}^{\infty} A_{i j} E_{2^{k+1} i+2^{k}-1,2^{k+1} j+2^{k}-1} .
$$

This map is well-defined because $(k, i) \mapsto 2^{k+1} i+2^{k}-1$ is one-to-one; Wagoner showed in [22, pp. 355] that it satisfies (5.3). Observe that the $x_{i}^{\prime} s$ and $y_{i}^{\prime} s$ are elements of $\Gamma(R)$. It is not hard to check, and noticed in [8, 4.8.2], that $\Phi(\Gamma(R)) \subset \Gamma(R)$, whence $\Gamma(R)$ is an infinite sum ring too. Now we remark that if $\mathfrak{A}$ is a bornological algebra, then

$$
\Gamma(\mathfrak{A}) \subset \Gamma^{\infty}(\mathfrak{A}) \subset \Gamma^{W}(\mathfrak{A})
$$

Furthermore, $\Phi$ also sends $\Gamma^{\infty}(\mathfrak{A})$ to itself. Thus if $\mathfrak{A}$ is unital, then $\Gamma^{\infty}(\mathfrak{A})$ is an infinite sum ring. We record this in the following proposition. 
Proposition 5.1 Let $\mathfrak{A}$ be a unital bornological algebra, and let $f_{i}$ be as in (5.4) and $\Phi$ as in (5.5) Then $\left(\Gamma^{\infty}(\mathfrak{A}), U_{f_{0}^{\dagger}}, U_{f_{1}^{\dagger}}, U_{f_{0}}, U_{f_{1}}, \Phi\right)$ is an infinite sum ring.

Corollary 5.2 Let $F: \mathbb{C}-\mathrm{Alg} \rightarrow \mathfrak{A b}$ be a functor. Assume that the restriction of $F$ to unital $\mathbb{C}$-algebras is split-exact and $M_{2}$-stable. Then $F\left(\Gamma^{\infty}(\mathfrak{A})\right)=0$ for any unital bornological algebra $\mathfrak{A}$. If furthermore $F$ is split exact on all $\mathbb{C}$-algebras, then $F\left(\Gamma^{\infty}(\mathfrak{A})\right)=0$ for any, not necessarily unital bornological algebra $\mathfrak{A}$.

Proof Immediate from Proposition 5.1 and [5, Proposition 2.3.1].

Example 5.3 Both Weibel's homotopy algebraic $K$-theory [23] and periodic cyclic homology [13] are $M_{2}$-stable and excisive on all $\mathbb{Q}$-algebras. Hence if $\mathfrak{A}$ is a bornological algebra, then

$$
K H_{*}\left(\Gamma^{\infty}(\mathfrak{A})\right)=H P_{*}\left(\Gamma^{\infty}(\mathfrak{A})\right)=0
$$

Algebraic $K$-theory groups $K_{n}$ are split exact and $M_{2}$ - stable for $n \leq 0$; the same is true of Karoubi-Villamayor $K$-groups $K V_{m}$ for $m \geq 1$ [18, Théorème 4.5]. Hence,

$$
K_{n}\left(\Gamma^{\infty}(\mathfrak{A})\right)=K V_{m}\left(\Gamma^{\infty}(\mathfrak{A})\right)=0 \quad(n \leq 0, m \geq 1)
$$

again for all $\mathfrak{A}$. For positive $n$, the groups $K_{n}$ are still split exact and $M_{2}$-stable on unital rings. The same is true of both the Hochschild and cyclic homology groups $H_{n}$ and $H C_{n}$ for $n \geq 0$; moreover these groups vanish for $n \leq-1$. Hence we have

$$
K_{n+1}\left(\Gamma^{\infty}(\mathfrak{A})\right)=H H_{n}\left(\Gamma^{\infty}(\mathfrak{A})\right)=H C_{n}\left(\Gamma^{\infty}(\mathfrak{A})\right)=0 \quad(n \geq 0)
$$

for any unital bornological algebra $\mathfrak{A}$.

\section{The algebra $\Gamma^{\infty}(\mathfrak{A})$ as a crossed product}

Let $2^{\mathbb{N}}$ denote the submonoid of idempotent elements of Emb

$$
2^{\mathbb{N}}=\left\{p: p \in \operatorname{Emb} p^{2}=p\right\} \subset \mathrm{Emb} .
$$

Note that if $p \in 2^{\mathbb{N}}$, then for $A=\operatorname{ran}(p)=\operatorname{dom}(p)$, we have $U_{p}=\operatorname{diag}\left(\chi_{A}\right)$, the diagonal matrix on the sequence

$$
\left(\chi_{A}\right)_{n}= \begin{cases}1 & n \in A \\ 0 & n \notin A\end{cases}
$$

We will often identify $p, U_{p}=\operatorname{diag}\left(\chi_{A}\right)$, and $\chi_{A}$. Notice that

$$
f_{*}(p) f=f p
$$


The subgroup of $\Gamma$ generated by the image of $2^{\mathbb{N}}$ under $f \mapsto U_{f}$ is the subring

$$
\mathcal{P}=\operatorname{span}\left\{U_{p}: p \in 2^{\mathbb{N}}\right\} \subset \Gamma \text {. }
$$

We also consider the monoid rings $\mathbb{Z}\left[2^{\mathbb{N}}\right]$ and $\mathbb{Z}[$ Emb], and the two-sided ideals

$$
\begin{aligned}
& I=\left\langle\left\{\chi_{A \sqcup B}-\chi_{A}-\chi_{B}: A, B \subset \mathbb{N}, \quad A \cap B=\emptyset\right\}\right\rangle \triangleleft \mathbb{Z}\left[2^{\mathbb{N}}\right], \\
& J=\left\langle\left\{\chi_{A \sqcup B}-\chi_{A}-\chi_{B}: A, B \subset \mathbb{N}, \quad A \cap B=\emptyset\right\}\right\rangle \triangleleft \mathbb{Z}[\text { Emb }] .
\end{aligned}
$$

Observe that $I$ and $J$ contain the element

$$
\chi_{A \cup B}-\chi_{A}-\chi_{B}+\chi_{A \cap B}
$$

for any pair of not necessarily disjoint subsets $A, B \subset \mathbb{N}$.

Lemma 6.1 i) $\mathcal{P}=\mathbb{Z}\left[2^{\mathbb{N}}\right] / I$.

ii) $\Gamma=\mathbb{Z}[\mathrm{Emb}] / J$

iii) If $\mathfrak{A}$ is a unital bornological algebra, then $\ell^{\infty}(\mathfrak{A}) \otimes_{\mathcal{P}} \Gamma \cong \Gamma^{\infty}(\mathfrak{A})$ as $\mathcal{P}$-bimodules.

Proof It is clear that there are natural surjective ring homomorphisms

$$
\begin{aligned}
& \pi_{1}: \mathbb{Z}\left[2^{\mathbb{N}}\right] / I \rightarrow \mathcal{P} \text { and } \\
& \pi_{2}: \mathbb{Z}[\mathrm{Emb}] / J \rightarrow \Gamma,
\end{aligned}
$$

and a natural surjective $\mathcal{P}$-bimodule homomorphism

$$
\pi_{3}: \ell^{\infty} \otimes_{\mathcal{P}} \Gamma \rightarrow \Gamma^{\infty}
$$

Let $\xi=\sum_{j=1}^{n} \lambda_{j} \chi_{A_{j}} \in \mathbb{Z}\left[2^{\mathbb{N}}\right]$ represent an element $\in$ ker $\pi_{1}$; for each subset $F \subset$ $\{1, \ldots, n\}$, let $A_{F}=\bigcap_{j \in F} A_{j} \cap \bigcap_{j \notin F} A_{j}^{c}$. From $\pi_{1}(\xi)_{\mid A_{F}}=0$ we get

$$
A_{F} \neq \emptyset \Rightarrow \sum_{j \in F} \lambda_{j}=0 .
$$

Next note that $\bigcup_{i=1}^{n} A_{i}=\sqcup_{F} A_{F}$; hence, modulo $I$, we have

$$
\begin{aligned}
\xi & \equiv \sum_{F} \sum_{j=1}^{n} \lambda_{j} \chi_{A_{j} \cap A_{F}} \\
& =\sum_{F}\left(\sum_{j \in F} \lambda_{j}\right) \chi_{A_{F}}=0 .
\end{aligned}
$$

This proves i). In order to prove ii) we have to show that $\operatorname{ker}\left(\pi_{2}\right)=0$. Let $\xi=$ $\sum_{j=1}^{n} \lambda_{j} f_{j} \in \mathbb{Z}[\mathrm{Emb}]$ be a representative of an element in $\operatorname{ker}\left(\pi_{2}\right)$. Let $A_{i}=\operatorname{dom} f_{i}$, 
and let $A_{F}$ be as above; then $\xi \equiv \sum_{F} \xi \chi_{A_{F}}$. Hence we may assume that the $A_{i}$ are disjoint. Furthermore, upon replacing $\xi$ by $\xi \chi_{A_{i}}$, and eliminating zero elements of Emb, we may assume that $A_{1}=\cdots=A_{n}$. For each $j \in \mathbb{N}$, we have

$$
\sum_{i=1}^{n} \lambda_{i} e_{f_{i}(j)}=0
$$

Let $K=\left\{f_{i}(j): i=1, \ldots, n\right\}$; for each $k \in K$, let $D_{k}=\left\{i: f_{i}(j)=k\right\}$. Then $D(j):=\left\{D_{k}\right\}_{k \in K}$ is a partition of $\{1, \ldots, n\}$, and $\sum_{i \in D_{k}} \lambda_{i}=0$. There is a finite set $\mathcal{D}$ of partitions arising in this way, since the number of all partitions of $\{1, \ldots, n\}$ is finite. For each $D \in \mathcal{D}$, let $J_{D}=\{j \in \mathbb{N}: D(j)=D\}$. Then $\sqcup_{D \in \mathcal{D}} J_{D}=\mathbb{N}$, and $\xi \equiv \sum_{D} \xi \cdot \chi_{D}$. Hence, upon replacing $\xi$ by $\xi \chi_{D}$ if necessary, we may assume that $\mathcal{D}$ has only one element $D=\left\{D_{1}, \ldots, D_{r}\right\}$. But $\xi \equiv \sum_{i} \chi_{D_{i}} \xi$, so we further reduce to the case when $r=1$. This means that $f_{1}=\cdots=f_{n}$ and, by (6.4), $\sum_{i} \lambda_{i} f_{i}$ is the zero element of $\mathbb{Z}[\mathrm{Emb}]$. We have proved ii). To prove iii) we must show that $\pi_{3}$ is injective. Let $\xi=\sum_{i=1}^{n} \alpha_{(i)} \otimes U_{f_{i}} \in \operatorname{ker} \pi_{3}$. Because

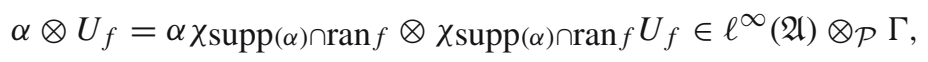

we may assume that $\operatorname{supp}\left(\alpha_{i}\right)=\operatorname{ran}\left(f_{i}\right)(i=1, \ldots, n)$. Proceeding as above, we may assume that $\operatorname{dom} f_{1}=\cdots=\operatorname{dom} f_{n}$. For each $j \in \mathbb{N}$, we have

$$
\sum_{i=1}^{n} \alpha_{(i)}(j) e_{f_{i}(j)}=0 .
$$

Proceeding as above again, we may reduce to the case $f_{1}=\cdots=f_{n}$. By (6.5), we have $\sum_{i=1}^{n} \alpha_{(i)}=0$. Thus

$$
\xi=\sum_{i=1}^{n} \alpha_{(i)} \otimes U_{f_{i}}=\left(\sum_{i=1}^{n} \alpha_{(i)}\right) \otimes U_{f_{1}}=0 .
$$

Remark 6.2 Given any monoid $M$, a representation of $M$ is the same thing as module over the monoid ring $\mathbb{Z}[M]$. In view of Lemma 6.1 , the modules over $\mathcal{P}$ and $\Gamma$ correspond to those representations of the inverse monoids $2^{\mathbb{N}}$ and Emb which are tight in the sense of Exel (see [15, Def. 13.1 and Prop. 11.9]).

Remark 6.3 It was proved in [8, Lemma 4.7.1] that the map

$$
\psi: \Gamma \otimes R \rightarrow \Gamma(R), \psi(A \otimes x)_{i, j}=A_{i j} x
$$

is an isomorphism. It follows from this that $\Gamma$ is flat as an abelian group. Therefore the map $J \otimes R \rightarrow \mathbb{Z}[\mathrm{Emb}] \otimes R$ is injective. Thus, by Lemma 6.1,

$$
\Gamma(R)=\mathbb{Z}[\mathrm{Emb}] \otimes R / J \otimes R=R[\mathrm{Emb}] / J R .
$$


Next observe that the inclusion $\mathcal{P} \subset \Gamma$ is a split injection. Indeed the map

$$
\Gamma \rightarrow \mathcal{P}, U_{f} \mapsto P_{\operatorname{dom} f}
$$

is a left inverse. It follows that if $R$ is any ring then the map $\psi: \mathcal{P} \otimes R \rightarrow \mathcal{P}(R):=$ $\psi(\mathcal{P} \otimes R)$ is an isomorphism. Thus using Lemma 6.1 and a similar argument as that given above for the case of $\Gamma$, one can show that

$$
\mathcal{P}(R)=R\left[2^{\mathbb{N}}\right] / I R \text {. }
$$

Because Emb is a monoid, if $\mathcal{A}$ is a ring on which Emb acts by ring endomorphisms we can form the crossed product $\mathcal{A} \#$ Emb. As an abelian group, $\mathcal{A} \#$ Emb $=\mathcal{A} \otimes \mathbb{Z}[\mathrm{Emb}]$ with multiplication given by

$$
(a \# f)(b \# g)=a f_{*}(b) \# f g \text {. }
$$

Here $\#=\otimes$ and $f_{*}(b)$ denotes the action of $f$ on Emb. Now assume that the Emb-ring $\mathcal{A}$ is also a $\mathcal{P}$-algebra, that is, it is a ring and a $\mathcal{P}$-bimodule, and these operations are compatible in the sense that

$$
(a p) b=a(p b)(a, b \in \mathcal{A}, p \in \mathcal{P})
$$

Further assume that $\mathcal{A}$ is central as a $\mathcal{P}$-bimodule, i.e. $p a=a p(a \in \mathcal{A}, p \in \mathcal{P})$, and that

$$
p a=p_{*}(a) \quad\left(p \in 2^{\mathbb{N}}\right) .
$$

Under all these conditions, we say that $\mathcal{A}$ is an Emb-bundle (cf. [1, Def. 2.10]). For $J \triangleleft \mathbb{Z}[\mathrm{Emb}]$ as in (6.3), we have

$$
\begin{aligned}
\mathcal{A} \# \mathrm{Emb} \triangleright \mathcal{A} \# J & =\operatorname{span}\{r \# j: r \in \mathcal{A}, j \in J\} \text { and } \\
\mathcal{A} \# \mathrm{Emb} \triangleright L & =\operatorname{span}\{r p \# h-r \# p h: r \in \mathcal{A}, p \in \mathcal{P}, h \in \mathrm{Emb}\} .
\end{aligned}
$$

Set

$$
\mathcal{A} \#_{\mathcal{P}} \Gamma=\mathcal{A} \# \mathrm{Emb} /(L+\mathcal{A} \# J) .
$$

Thus, $\mathcal{A \#}_{\mathcal{P}} \Gamma=\mathcal{A} \otimes_{\mathcal{P}} \Gamma$ as left $\mathcal{P}$-modules, and the product is that induced by (6.6); we have

$$
\left(a \# U_{f}\right)\left(b \# U_{g}\right)=a f_{*}(b) \# U_{f g} \in \mathcal{A \#}_{\mathcal{P}} \Gamma .
$$

Proposition 6.4 Let $\mathfrak{A}$ be a bornological algebra. The map

$$
\ell^{\infty}(\mathfrak{A}) \#_{\mathcal{P}} \Gamma \rightarrow \Gamma^{\infty}(\mathfrak{A}), \quad \alpha \# U_{f} \mapsto \operatorname{diag}(\alpha) U_{f}
$$


is an isomorphism of $\mathcal{P}$-algebras. If $S \triangleleft \ell^{\infty}$ is a symmetric ideal, then (6.9) sends $S(\mathfrak{A}) \#_{\mathcal{P}} \Gamma$ isomorphically onto $I_{S(\mathfrak{A})} \triangleleft \Gamma^{\infty}(\mathfrak{A})$.

Proof Assume first that $\mathfrak{A}$ is unital. Then the map (6.9) is the same as that of Lemma 6.1(iii). Hence, it is bijective. By (3.1) and (6.8), it is an algebra homomorphism. This proves the first assertion in the unital case; the second is immediate from the fact that (6.9) is bijective and maps $S(\mathfrak{A}) \#_{\mathcal{P}} \Gamma$ onto $I_{S(\mathfrak{A})}$. For not necessarily unital $\mathfrak{A}$, write $\tilde{\mathfrak{A}}$ for its unitalization as a bornological algebra. We have an exact sequence

$$
0 \rightarrow S(\mathfrak{A}) \rightarrow S(\tilde{\mathfrak{A}}) \rightarrow S \rightarrow 0
$$

Observe that the inclusion $\mathbb{C} \subset \tilde{\mathfrak{A}}$ induces a $\mathcal{P}$-module homomorphism $S \rightarrow S(\tilde{\mathfrak{A}})$ which splits the sequence (6.10). Hence we get an exact sequence

$$
0 \rightarrow S(\mathfrak{A}) \#_{\mathcal{P}} \Gamma \rightarrow S(\tilde{\mathfrak{A}}) \#_{\mathcal{P}} \Gamma \rightarrow S \#_{\mathcal{P}} \Gamma \rightarrow 0 .
$$

Combining this sequence with the unital case of the proposition, we obtain an isomorphism

$$
S(\mathfrak{A}) \#_{\mathcal{P}} \Gamma \stackrel{\cong}{\longrightarrow} \operatorname{ker}\left(I_{S(\mathfrak{A})} \rightarrow I_{S}\right)=I_{S(\mathfrak{A})}
$$

\section{Homotopy invariance}

\subsection{Crossed products by the Cohn ring}

The following two elements of Emb will play a central role in what follows

$$
\begin{aligned}
& s_{i}: \mathbb{N} \rightarrow \mathbb{N} \quad(i=1,2) \\
& s_{i}(m)=2 m+i-1 .
\end{aligned}
$$

We have the following relations

$$
s_{i}^{\dagger} s_{j}=\delta_{i, j} \quad i=1,2
$$

Following standard conventions, if $v$ is a word of length $l$ on $\{1,2\}$, we write $s_{v}=$ $s_{\nu_{1}} \cdots s_{\nu_{l}}$ and $s_{\nu}^{\dagger}=\left(s_{\nu}\right)^{\dagger}$. Thus for the empty word we have $s_{\emptyset}=s_{\emptyset}^{\dagger}=1$. Observe that if $\mu$ is of length $l$ then

$$
s_{\mu}(n)=2^{l} n+\sum_{i=1}^{l}\left(\mu_{i}-1\right) 2^{i-1} .
$$


Put

$$
W_{2}^{l}=\{\text { words of length } l \text { on }\{1,2\}\}, \quad W_{2}=\bigcup_{l=0}^{\infty} W_{2}^{l} \text {. }
$$

We write

$$
\mathcal{M}_{2}=\left\{s_{\mu}\left(s_{\nu}\right)^{\dagger}: \mu, v \in W_{2}\right\}
$$

Thus $\mathcal{M}_{2} \subset$ Emb is the inverse submonoid generated by the $s_{i}$. Its idempotent submonoid is

$$
E\left(\mathcal{M}_{2}\right)=\left\{s_{v}\left(s_{v}\right)^{\dagger}: v \in W_{2}\right\}
$$

One checks, using (7.2) that $s_{\mu} s_{v}^{\dagger}=s_{\mu^{\prime}} s_{v^{\prime}}^{\dagger}$ if and only if $\mu=\mu^{\prime}$ and $v=v^{\prime}$. It follows that $\mathcal{M}_{2}$ is the universal inverse monoid on generators $s_{1}, s_{2}$ subject to the relations (7.1). Write

$$
C_{2}=\mathbb{Z}\left[\mathcal{M}_{2}\right] \supset \mathcal{P}_{2}=\mathbb{Z}\left[E\left(\mathcal{M}_{2}\right)\right]
$$

The ring $C_{2}$ is the Cohn ring on two generators [3]; it is a purely algebraic version of the Toeplitz Cuntz algebra (called $\mathcal{E}_{2}$ in [11]). The assignment

$$
E_{s_{\mu}(1), s_{v}(1)} \mapsto s_{\mu}\left(1-\sum_{i=1}^{2} s_{i} s_{i}^{\dagger}\right) s_{v}^{*} .
$$

defines an isomorphism between $M_{\infty}$ and the ideal of $C_{2}$ generated by $1-\sum_{i=1}^{2} s_{i} s_{i}^{\dagger}$. We shall identify each element of $M_{\infty}$ with its image in $C_{2}$. If $\mathfrak{A}$ is a bornological algebra and $S \triangleleft \ell^{\infty}$ is a symmetric ideal, then we can consider the action of $\mathcal{M}_{2}$ on $S(\mathfrak{A})$ coming from restriction of the Emb action, and form the crossed product $S(\mathfrak{A}) \# \mathcal{M}_{2}$. Recall from Sect. 6 that $S(\mathfrak{A}) \# \mathcal{M}_{2}=S(\mathfrak{A}) \otimes_{\mathbb{Z}} \mathbb{Z}\left[\mathcal{M}_{2}\right]$ equipped with the product (6.6). Put

$$
S(\mathfrak{A}) \#_{\mathcal{P}_{2}} C_{2}=S(\mathfrak{A}) \# \mathcal{M}_{2} /\left\langle\alpha p \# f-\alpha \# p f: p \in E\left(\mathcal{M}_{2}\right), f \in \mathcal{M}_{2}\right\rangle .
$$

As a vector space, $S(\mathfrak{A}) \#_{\mathcal{P}_{2}} C_{2}=S(\mathfrak{A}) \otimes_{\mathcal{P}_{2}} C_{2}$; the product is defined as in (6.6). We have an algebra homomorphism

$$
\rho: S(\mathfrak{A}) \#_{\mathcal{P}_{2}} C_{2} \rightarrow I_{S(\mathfrak{A})}, \rho(\alpha \# f)=\operatorname{diag}(\alpha) U_{f}
$$

Lemma 7.1 The map (7.3) is injective.

Proof Any nonzero element $x \in C_{2}$ can be written as a finite sum of nonzero terms

$$
x=\sum_{\mu, v} \alpha(\mu, v) \# s_{\mu} s_{v}^{\dagger}
$$


Let $l$ be the maximum length of all the multi-indices $\mu$ appearing in the expression above. Remark that we may rewrite (7.4) as another finite sum

$$
x=\sum_{i, j} x_{i, j} \# E_{i, j}+\sum_{l(\mu)=l} \beta_{\mu, \nu} \# s_{\mu} s_{v}^{\dagger} .
$$

such that

$$
x_{i, j} \neq 0 \Rightarrow i<2^{l} \text {. }
$$

Indeed this follows from (7.2) and from the identities

$$
\begin{aligned}
s_{\mu} s_{v}^{\dagger} & =s_{\mu}\left(1-\sum_{i=1}^{2} s_{i} s_{i}^{\dagger}\right) s_{v}^{\dagger}+\sum_{i=1}^{2} s_{\mu i} s_{v i}^{\dagger} \\
& =E_{\mu(1), v(1)}+\sum_{i=1}^{2} s_{\mu i} s_{v i}^{\dagger} .
\end{aligned}
$$

Suppose that the element (7.5) is in $\operatorname{ker} \rho$. Observe that $\rho\left(\chi_{\{i\}} \otimes E_{i, j}\right)=E_{i, j}$. Hence, we have

$$
0=\sum_{i, j} x_{i, j} E_{i, j}+\sum_{l(\mu)=l, v} \operatorname{diag}\left(\beta_{\mu, \nu}\right) U_{s_{\mu}} U_{s_{v}}^{*}
$$

But by (7.2), for $\mu$ as in (7.7), we have

$$
\operatorname{ran}\left(U_{s_{\mu}} U_{s_{\nu}}^{*}\right)=\operatorname{span}\left\{e_{n}: n=2^{l} m+\sum_{i=1}^{l}\left(\mu_{i}-1\right) 2^{i-1} m \in \mathbb{N}\right\} .
$$

This together with (7.6) imply that each of the summands of (7.7) vanishes. Thus

$$
x_{i, j}=0 \text { and } \operatorname{diag}\left(\beta_{\mu, v}\right) U_{s_{\mu}} U_{s_{v}}^{*}=0
$$

for all $i, j$ and all $\mu$ and $v$ in (7.6). Hence,

$$
\emptyset=\operatorname{supp} \beta_{\mu, \nu} \cap\left(2^{l} \mathbb{N}+\sum_{i=1}^{l}\left(\mu_{i}-1\right) 2^{i-1}\right)=\operatorname{supp}\left(s_{\mu} s_{\mu}^{\dagger}\right)_{*}\left(\beta_{\mu, \nu}\right) .
$$

It follows that $\beta_{\mu, \nu} \# s_{\mu} s_{v}^{\dagger}=0$ and therefore the element (7.5) must be zero, finishing the proof.

Remark 7.2 Let $S \triangleleft \ell^{\infty}$ be a nonzero symmetric ideal and let $c_{f}$ be as in Example 3.8. Then $S$ contains $c_{f}$ and thus if we identify $S \#_{\mathcal{P}_{2}} C_{2}$ with its image in $I_{S}$, we have

$$
I_{S} \supset S \#_{\mathcal{P}_{2}} C_{2} \supset c_{f} \#_{\mathcal{P}_{2}} C_{2}=M_{\infty}
$$


In particular the completion of $c_{0} \#_{\mathcal{P}_{2}} C_{2}$ with respect to the operator norm in $\mathcal{B}\left(\ell^{2}\right)$ coincides with the completion of $M_{\infty} \mathbb{C}$ and of $I_{c_{0}}$; it is the ideal $\mathcal{K}=J_{c_{0}}$ of compact operators. Similarly, for $p \geq 1$ the completion of $\ell^{p} \#_{\mathcal{P}_{2}} C_{2}$ for the $p$-Schatten norm $\|T\|_{p}=\operatorname{Tr}\left(|T|^{p}\right)$ coincides with that of $I_{\ell^{p}}$; it is the Schatten ideal $\mathcal{L}^{p}$.

\subsection{The Cohn ring and homotopy invariance}

Let $\mathbb{V}$ be a bornological vector space, $T$ a compact Hausdorff topological space, $X$ a metric space, and $1 \geq \lambda>0$. Put

$$
\begin{aligned}
C(T, \mathbb{V}) & =\{f: T \rightarrow \mathbb{V} \text { continuous }\} \\
H^{\lambda}(X, \mathbb{V}) & =\{f: X \rightarrow \mathbb{V} \lambda-\text { Hölder continuous }\} .
\end{aligned}
$$

We refer the reader to [12, §2.1.1 and $\$ 3.1 .4]$ for the definitions of continuity and Hölder continuity in the bornological setting, as well as for those of the canonical uniform bornologies that the above algebras carry.

Let $S \triangleleft \ell^{\infty}$ be a symmetric ideal and $\mathfrak{A}$ a bornological algebra. We have a natural inclusion

$$
\text { inc : } \mathfrak{A} \subset S(\mathfrak{A}), a \mapsto(a, 0,0, \ldots) \text {. }
$$

Lemma 7.3 (cf. [12, Lemma 3.26]) Let $F: \mathbb{C}-\mathrm{Alg} \rightarrow \mathfrak{A b}$ be a split-exact, $M_{2}$ stable functor, $\mathfrak{B}$ a bornological algebra, $\mathrm{ev}_{t}: C([0,1], \mathfrak{B}) \rightarrow \mathfrak{B}$ the evaluation map, and $0<\lambda \leq 1$.

i)

$$
F\left(C([0,1], \mathfrak{B}) \stackrel{\mathrm{ev}_{t}}{\rightarrow} \mathfrak{B} \stackrel{\text { inc }}{\rightarrow} c_{0}(\mathfrak{B}) \stackrel{-\# 1}{\rightarrow} c_{0}(\mathfrak{B}) \#_{\mathcal{P}_{2}} C_{2}\right)
$$

is independent of $t$.

ii) If $p>1 / \lambda$, then

$$
F\left(H^{\lambda}([0,1], \mathfrak{B}) \stackrel{\mathrm{ev}_{t}}{\rightarrow} \mathfrak{B} \stackrel{\text { inc }}{\rightarrow} \ell^{p}(\mathfrak{B}) \stackrel{-\# 1}{\rightarrow} \ell^{p}(\mathfrak{B}) \#_{\mathcal{P}_{2}} C_{2}\right)
$$

is independent of $t$.

Proof Let $S$ be either $c_{0}$ or $\ell^{p}$. In the first case, put $\mathfrak{B}[0,1]=C([0,1], \mathfrak{B})$; in the second, let $\lambda>1 / p$ and set $\mathfrak{B}[0,1]=H^{\lambda}([0,1], \mathfrak{B})$. Let

$$
\mathbb{Z}_{\geq 0} \times \mathbb{Z}_{\geq 0} \supset X=\left\{(l, k): k \leq 2^{l}-1\right\}
$$

Let $\phi_{+}, \phi_{-}, \phi_{0}^{2}$ and $\phi_{-}^{2}$ be the homomorphisms $\mathfrak{B}[0,1] \rightarrow \ell^{\infty}(X, \mathfrak{B})$ defined in the proof of [12, Lemma 3.26]. One checks that $\left(\phi_{+}, \phi_{-}\right)$and $\left(\phi_{0}^{2}, \phi_{-}^{2}\right)$ are quasihomomorphisms $\mathfrak{B}[0,1] \rightarrow S(X, \mathfrak{B})$. Furthermore, it is shown in loc. cit. that there 
are elements $V, \bar{V} \in \operatorname{Emb}(X)$ such that for

$$
\operatorname{inc}_{0,0}: \mathfrak{B} \rightarrow S(X, \mathfrak{B}), \operatorname{inc}_{0,0}(a)_{l, k}=a \delta_{l, 0} \delta_{k, 0}
$$

we have

$$
\begin{aligned}
F\left(\mathrm{inc}_{0,0} \circ \mathrm{ev}_{0}\right)-F\left(\mathrm{inc}_{0,0} \circ \mathrm{ev}_{1}\right)= & \left(F\left(\bar{V}_{*}\right)-1\right) F\left(\phi_{-}, \phi_{+}\right) \\
& +\left(F\left(V_{*}\right)-1\right) F\left(\phi_{0}^{2}, \phi_{-}^{2}\right) .
\end{aligned}
$$

Consider the bijection $\psi: X \rightarrow \mathbb{N}$

$$
\psi(l, k)=2^{l}+k .
$$

Let $s_{1}, s_{2}$ be the generators (7.1) of $C_{2}$. Let $v, \bar{v} \in$ Emb be the conjugates of $V$ and $\bar{V}$ under $\psi$. One checks that, for $\rho$ as in (7.3), we have

$$
\begin{aligned}
\bar{v} & =s_{2} \text { and } \\
U_{v} & =\rho\left(1-s_{1} s_{1}^{\dagger}-s_{2} s_{2}^{\dagger}+s_{2} s_{1}^{\dagger}+s_{1} s_{2}^{\dagger}\right)
\end{aligned}
$$

Now recall that $C_{2}=\mathbb{Z}\left[\mathcal{M}_{2}\right]$ and write $*: C_{2} \rightarrow C_{2}$ for the involution induced by $\dagger$. It follows from (7.11) that the element

$$
C_{2} \ni f=1-s_{1} s_{1}^{\dagger}-s_{2} s_{2}^{\dagger}+s_{2} s_{1}^{\dagger}+s_{1} s_{2}^{\dagger}
$$

satisfies $f^{*} f=1$. Hence if $g$ is any of $1 \# s_{2}, 1 \# f \in \ell^{\infty}(\tilde{\mathfrak{B}}) \# C_{2}$, we have an algebra homomorphism

$$
\operatorname{conj}(g): S(\mathfrak{B}) \# C_{2} \rightarrow S(\mathfrak{B}) \# C_{2}, x \mapsto g x g^{*}
$$

Moreover, because $F$ is $M_{2}$-stable by assumption and $S(\mathfrak{B}) \# C_{2}$ is an ideal in $\ell^{\infty}(\tilde{\mathfrak{B}}) \# C_{2}, F(\operatorname{conj}(g))$ is the identity [5, Proposition 2.2.6]. Let $\phi_{0}^{\prime 2}, \phi_{-}^{\prime 2}, \phi_{+}^{\prime}$ and $\phi_{-}^{\prime}$ be the maps $\mathfrak{B}[0,1] \rightarrow S(\mathfrak{B})$ obtained from $\phi_{0}^{2}, \phi_{-}^{2}, \phi_{+}$, and $\phi_{-}$after conjugating with $U_{\psi}$. Then (7.8) gives the identity

$$
\begin{aligned}
\left.F\left((\operatorname{inc~ev})_{0}\right) \# 1\right)-F\left(\left(\operatorname{inc~ev}_{1}\right) \# 1\right)= & \left(F\left(\operatorname{conj}\left(1 \# s_{2}\right)\right)-1\right) F\left(\phi_{-}^{\prime}, \phi_{+}^{\prime}\right) \\
& +(F(\operatorname{conj}(1 \# f))-1) F\left(\phi_{0}^{2}, \phi_{-}^{2}\right)=0 .
\end{aligned}
$$

We have proved that $F\left(\left(\right.\right.$ inc $\left.\left.\circ \mathrm{ev}_{0}\right) \# 1\right)=F\left(\left(\mathrm{inc} \circ \mathrm{ev}_{1}\right) \# 1\right)$. The proposition now follows from the fact that if $t \in[0,1]$ then $\mathrm{ev}_{t}$ and $\mathrm{ev}_{0}$ are linearly homotopic.

Remark 7.4 The key property of $C_{2}$ used in the proof of Lemma 7.3 is that it contains the elements (7.10) and (7.12). In fact it is not hard to check that they generate $C_{2}$ as a ring. Hence taking crossed product with $C_{2}$ may be regarded as the smallest construction which makes the proof given above work. 
Remark 7.5 If $\mathfrak{A}$ is a $C^{*}$-algebra, then $c_{0}(\mathfrak{A})=c_{0} \widetilde{\otimes} \mathfrak{A}$ is the spatial $C^{*}$-algebra tensor product. The inclusion $c_{0} \subset I_{c_{0}} \subset \mathcal{K}$ is equivariant for the action of Emb, and so we get a map $c_{0}(\mathfrak{A}) \#_{\mathcal{P}_{2}} C_{2} \rightarrow \mathfrak{A} \otimes \mathcal{X}$. Composing the latter with the inclusion $\mathfrak{A} \rightarrow c_{0}(\mathfrak{A}) \#_{\mathcal{P}_{2}} C_{2}$ of Lemma 7.3 we obtain the map $\iota: \mathfrak{A} \rightarrow \mathfrak{A} \widetilde{\otimes} \mathcal{K}, a \mapsto a \tilde{\otimes} E_{1,1}$. Hence, the lemma implies that if $F: \mathbb{C}-\mathrm{Alg} \rightarrow \mathfrak{A b}$ is split-exact and $M_{2}$-stable, then, for every $C^{*}$-algebra $\mathfrak{B}$, the map

$$
F\left(C([0,1], \mathfrak{B}) \stackrel{\mathrm{ev}_{t}}{\rightarrow} \mathfrak{B} \stackrel{\iota}{\rightarrow} \mathfrak{B} \widetilde{\otimes} \mathcal{K}\right)
$$

is independent of $t$. One can use this to prove that $F$ is homotopy invariant on stable $C^{*}$-algebras, thus obtaining a weak version of Higson's homotopy invariance theorem [17, Theorem 3.2.2]. Indeed it suffices to show that $F(\iota)$ is injective if $\mathfrak{B}=\mathfrak{A} \tilde{\otimes} \mathcal{K}$, and this follows from the fact that there is a map $\mathcal{K} \otimes \mathcal{K} \rightarrow M_{2} \mathcal{K}$ (in fact an isomorphism) such that the following diagram commutes

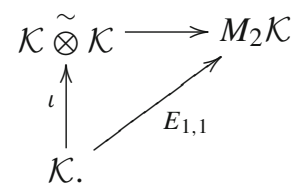

Next suppose that $\mathfrak{B}$ is any bornological algebra. Write $\hat{\otimes}$ for the projective tensor product. For each $p \geq 1$ we have a map $\ell^{p} \hat{\otimes} \mathfrak{B} \rightarrow \ell^{p}(\mathfrak{B})$. This map is an isomorphism if $p=1$; using this isomorphism as above, we obtain a map

$$
\ell^{1}(\mathfrak{A}) \#_{\mathcal{P}_{2}} C_{2} \rightarrow \mathfrak{A} \hat{\otimes} \mathcal{L}^{1}
$$

In general $\ell^{p} \hat{\otimes} \mathfrak{A} \rightarrow \ell^{p}(\mathfrak{A})$ is not an isomorphism. Note, however, that for every $p \geq 1$, the quotient $\ell^{p}(\mathfrak{A}) / \ell^{1}(\mathfrak{A})$ is a nilpotent ring. Assume that the functor $F$ is strongly nilinvariant in the sense that if $f: A \rightarrow B$ is a homomorphism with nilpotent kernel, and such that $f(A) \triangleleft B$ and $B / f(A)$ is nilpotent, then $F(f)$ is an isomorphism. Then $F\left(\ell^{1}(\mathfrak{A}) \#_{\mathcal{P}_{2}} C_{2}\right) \rightarrow F\left(\ell^{p}(\mathfrak{A}) \#_{\mathcal{P}_{2}} C_{2}\right)$ and $F\left(\mathfrak{A} \hat{\otimes} \mathcal{L}^{1}\right) \rightarrow F\left(\mathfrak{A} \hat{\otimes} \mathcal{L}^{p}\right)$ are isomorphisms for all $p \geq 1$. Moreover we also have a commutative diagram

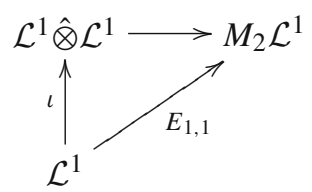

Let BAlg be the category of bornological algebras and bounded homomorphisms. Using Lemma 7.3 together with diagram (7.14) above and proceeding as before, one shows that if $F$ is split-exact, $M_{2}$-stable, and strongly nilinvariant, then the functor

$$
\mathrm{BAlg} \rightarrow \mathfrak{A} \mathfrak{b}, \mathfrak{A} \mapsto F\left(\mathfrak{A} \hat{\otimes} \mathcal{L}^{1}\right)
$$


is invariant under Hölder-continuous homotopies. This gives a (weak) bornological version of [9, Theorem 6.1.6]. Observe that the stability properties (7.13) and (7.14) play a crucial role in the arguments above. We do not have an analogous stability result for the uncompleted algebras $c_{0}(\mathfrak{A}) \#_{\mathcal{P}_{2}} C_{2}$ and $\ell^{1}(\mathfrak{A}) \#_{\mathcal{P}_{2}} C_{2}$. In the next subsection we shall prove a version of stability for crossed products with $\Gamma$. This will enable us to prove a homotopy invariance theorem in the following subsection.

\subsection{Stability}

Lemma 7.6 i) There is a natural isomorphism $\Gamma(\mathbb{N} \sqcup \mathbb{N}) \cong M_{2} \Gamma$.

ii) Let $\mathfrak{A}$ be a bornological algebra and $S \triangleleft \ell^{\infty}$ a symmetric ideal. Then $I_{S(\mathbb{N} \cup \mathbb{N}, \mathfrak{A})} \cong$ $M_{2} I_{S(\mathfrak{A})}$.

Proof Let $p_{1}, p_{2} \in \operatorname{Emb}(\mathbb{N} \sqcup \mathbb{N})$ be the inclusions of each of the copies of $\mathbb{N}$. One checks that the map

$$
\phi: \Gamma(\mathbb{N} \sqcup \mathbb{N}) \rightarrow M_{2} \Gamma, \quad \phi(a)_{i j}=U_{p_{i}} a U_{p_{j}}
$$

is an isomorphism. To prove part ii) one checks that the following composite of isomorphisms of abelian groups is a homomorphism of algebras

$$
\begin{aligned}
S(\mathbb{N} \sqcup \mathbb{N}, \mathfrak{A}) \#_{\mathcal{P}(\mathbb{N} \sqcup \mathbb{N})} \Gamma(\mathbb{N} \sqcup \mathbb{N}) & \cong(S(\mathfrak{A}) \oplus S(\mathfrak{A})) \otimes_{\mathcal{P} \oplus \mathcal{P}} M_{2}(\Gamma) \\
& \cong M_{2}\left(S(\mathfrak{A}) \#_{\mathcal{P}} \Gamma\right) .
\end{aligned}
$$

Let $\mathfrak{A}$ be a bornological algebra and let $\iota: \ell^{\infty}(\mathfrak{A}) \rightarrow \ell^{\infty}(\mathbb{N} \times \mathbb{N}, \mathfrak{A})$ be the inclusion

$$
\iota(\alpha)(m, n)=\alpha(m) \delta_{1, n} .
$$

Also let $S \triangleleft \ell^{\infty}$ be a symmetric ideal; put

$$
\begin{aligned}
& J: S(\mathfrak{A}) \#_{\mathcal{P}} \Gamma \rightarrow S(\mathbb{N} \times \mathbb{N}, \mathfrak{A}) \#_{\mathcal{P}(\mathbb{N} \times \mathbb{N})} \Gamma(\mathbb{N} \times \mathbb{N}) \\
& J\left(\alpha \# U_{f}\right)=\iota(\alpha) \#\left(U_{f \times \chi\{1\}}\right) .
\end{aligned}
$$

Proposition 7.7 Let $\mathfrak{A}$ be a bornological algebra and $S \triangleleft \ell^{\infty}$ a symmetric ideal. Then any $M_{2}$-stable functor $F: \mathbb{C}-\mathrm{Alg} \rightarrow \mathfrak{A b}$ sends the map $\mathrm{J}$ of (7.15) to an isomorphism.

Proof Choose a bijection $\mathbb{N} \times \mathbb{N} \rightarrow \mathbb{N} \sqcup \mathbb{N}$ sending $\mathbb{N} \times\{1\}$ bijectively onto the first copy of $\mathbb{N}$. This bijection induces an isomorphism

$$
S(\mathbb{N} \times \mathbb{N}, \mathfrak{A}) \#_{\mathcal{P}(\mathbb{N} \times \mathbb{N})} \Gamma(\mathbb{N} \times \mathbb{N}) \stackrel{\cong}{\longrightarrow} S(\mathbb{N} \sqcup \mathbb{N}, \mathfrak{A}) \#_{\mathcal{P}(\mathbb{N} \sqcup \mathbb{N})} \Gamma(\mathbb{N} \sqcup \mathbb{N})
$$


Composing this map with the isomorphism of Lemma 7.6, we obtain an isomorphism which fits into a commutative diagram

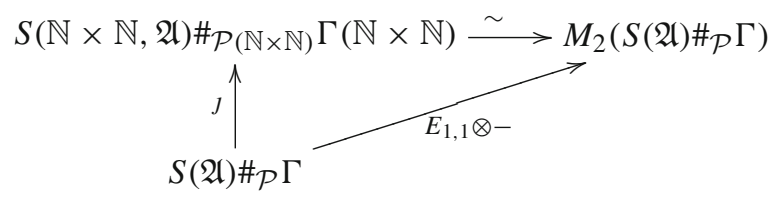

This concludes the proof.

7.4 A homotopy invariance theorem

Let $f_{0}, f_{1}: \mathfrak{A} \rightarrow \mathfrak{B}$ be homomorphisms of bornological algebras and $0<\lambda \leq 1$. A $\lambda$-Hölder continuous homotopy from $f_{0}$ to $f_{1}$ is a homomorphism $H: \mathfrak{A} \rightarrow$ $H^{\lambda}([0,1], \mathfrak{B})$ such that $\mathrm{ev}_{i} H=f_{i}(i=0,1)$. We say that a functor $F:$ BAlg $\rightarrow \mathfrak{A b}$ is invariant under $\lambda$-Hölder homotopies if it maps $\lambda$-Hölder homotopic homomorphisms to equal maps.

Theorem 7.8 Let $F: \mathbb{C}-\mathrm{Alg} \rightarrow \mathfrak{A} \mathfrak{b}$ be a split-exact, $M_{2}$-stable functor.

i) The functor

$$
\mathrm{BAlg} \rightarrow \mathfrak{A} \mathfrak{b}, \mathfrak{B} \mapsto F\left(I_{c_{0}(\mathfrak{B})}\right)
$$

is invariant under continuous homotopies.

ii) If $1 \geq \lambda>0$ and $p>1 / \lambda$, then the functor

$$
\mathrm{BAlg} \rightarrow \mathfrak{A} \mathfrak{b}, \mathfrak{B} \mapsto F\left(I_{\ell^{p}}(\mathfrak{B})\right)
$$

is invariant under $\lambda$-Hölder homotopies.

Proof Let $\mathfrak{A}$ be a bornological algebra. We adopt the notation of the proof of Lemma 7.3. Thus $S$ will be either $c_{0}$ or $\ell^{p}$, and $\mathfrak{A}[0,1]$ will stand for $C([0,1], \mathfrak{A})$ in the first case, and for $H^{\lambda}([0,1], \mathfrak{A})$ in the second. By the argument of the proof of Lemma 7.3 applied to the functor

$$
G=F\left(S(-) \#_{\mathcal{P}} \Gamma\right),
$$

we have the following identity

$$
\begin{aligned}
\left.G(\operatorname{inc})\left(G\left(\mathrm{ev}_{0}\right)\right)-G\left(\mathrm{ev}_{1}\right)\right)= & \left(G\left(\left(s_{2}\right)_{*}\right)-1\right) G\left(\phi_{-}^{\prime}, \phi_{+}^{\prime}\right) \\
& +\left(G\left(f_{*}\right)-1\right) G\left(\phi_{0}^{\prime 2}, \phi_{-}^{\prime 2}\right) .
\end{aligned}
$$

Now if $h \in$ Emb then $G\left(h_{*}\right)$ is the result of applying $F$ to the map

$$
S\left(h_{*}\right) \#_{\mathcal{P}} \Gamma: S(S(\mathfrak{A})) \#_{\mathcal{P}} \Gamma \rightarrow S(S(\mathfrak{A})) \#_{\mathcal{P}} \Gamma .
$$


Here the crossed product is taken with respect to the action on the external $S$. In addition, we consider the action of $\Gamma$ on the inner $S$ and take the crossed product again; we write $\left(S(S(\mathfrak{A}))_{\mathcal{P}} \Gamma\right) \#_{\mathcal{P}} \Gamma$ for the resulting algebra. We have an inclusion

$$
\text { inc }^{\prime}=-\# 1: S(S(\mathfrak{A})) \#_{\mathcal{P}} \Gamma \subset\left(S(S(\mathfrak{A})) \#_{\mathcal{P}} \Gamma\right) \#_{\mathcal{P}} \Gamma
$$

and a commutative diagram

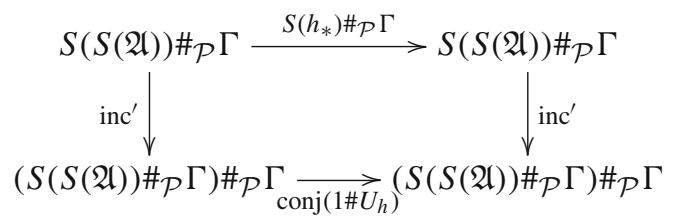

Because $F$ is $M_{2}$-stable, $F\left(\operatorname{conj}\left(1 \# U_{h}\right)\right)$ is the identity map, since

$$
\left.S(S(\mathfrak{A})) \#_{\mathcal{P}} \Gamma\right) \#_{\mathcal{P}} \Gamma \triangleleft\left(\ell^{\infty}\left(\ell^{\infty}(\mathfrak{A})\right) \#_{\mathcal{P}} \Gamma\right) \#_{\mathcal{P}} \Gamma \ni 1 \# U_{h} .
$$

Hence, by (7.17),

$$
\begin{aligned}
& \left.F\left(\mathrm{inc}^{\prime}(S(\mathrm{inc}) \# \Gamma)\right)\right)\left(F\left(S\left(\mathrm{ev}_{0}\right) \# \Gamma\right)-F\left(S\left(\mathrm{ev}_{1}\right) \# \Gamma\right)\right. \\
& \quad=F\left(\mathrm{inc}^{\prime}\right)\left(G\left(\left(s_{2}\right)_{*}\right)-1\right) G\left(\phi_{-}^{\prime}, \phi_{+}^{\prime}\right)+F\left(\mathrm{inc}^{\prime}\right)\left(G\left(f_{*}\right)-1\right) G\left(\phi_{0}^{\prime 2}, \phi_{-}^{\prime 2}\right)=0 .
\end{aligned}
$$

We have to show that

$$
F\left(\text { inc }^{\prime}(S(\text { inc }) \# \Gamma)\right)
$$

is injective. Observe that we have a natural isomorphism

$$
\mu: S(S(\mathfrak{A})) \stackrel{\cong}{\longrightarrow} S(\mathbb{N} \times \mathbb{N}, \mathfrak{A}), \quad \mu(\alpha)_{m, n}=(\alpha(n))(m) .
$$

For $h \in$ Emb the isomorphism (7.20) transforms $S\left(h_{*}\right)$ into the action of $1 \times h \in$ $\operatorname{Emb}(\mathbb{N} \times \mathbb{N})$, and $h_{*} S$ into that of $h \times 1$. Hence we have a map

$$
\begin{aligned}
& \operatorname{inc}^{\prime \prime}:\left(S(S(\mathfrak{A})) \#_{\mathcal{P}} \Gamma\right) \#_{\mathcal{P}} \Gamma \rightarrow S(\mathbb{N} \times \mathbb{N}) \#_{\mathcal{P}(\mathbb{N} \times \mathbb{N})} \Gamma(\mathbb{N} \times \mathbb{N}) \\
& \operatorname{inc}^{\prime \prime}\left(\alpha \# U_{g} \# U_{h}\right)=\mu(\alpha) \#\left(U_{g \times h}\right)
\end{aligned}
$$

Observe that the composite

$$
\text { inc }^{\prime \prime} \text { inc }^{\prime}(S(\text { inc }) \# \Gamma)=J
$$

is the map of (7.15). By Proposition 7.7, this implies that the map (7.19) is injective, concluding the proof. 


\section{$8 K$-theory}

8.1 Homotopy algebraic $K$-theory

Let $0<p \leq \infty$. Put

$$
\ell^{p-}=\bigcup_{q<p} \ell^{q}
$$

For $0<p<\infty$ we also consider

$$
\ell^{p+}=\bigcap_{q>p} \ell^{q}
$$

We say that a functor $F:$ BAlg $\rightarrow \mathfrak{A} \mathfrak{b}$ is Hölder homotopy invariant if it is invariant under $\lambda$-Hölder homotopies for all $0<\lambda \leq 1$. Recall from [12, §2] that a bornological algebra is called a local Banach algebra if it is a filtering union of Banach subalgebras. Similarly we say that a bornological algebra is a bornolocal $C^{*}$-algebra if it is a filtering union of $C^{*}$-subalgebras. If $\mathfrak{A}=\cup_{\lambda} \mathfrak{A}_{\lambda}$ and $\mathfrak{B}=\cup_{\mu} \mathfrak{B}_{\mu}$ are bornolocal $C^{*}$-algebras, we define their spatial tensor product as the algebraic colimit of the spatial tensor products $\mathfrak{A}_{\lambda} \widetilde{\otimes} \mathfrak{B}_{\mu} ; \mathfrak{A} \otimes \mathfrak{B}=\operatorname{colim}_{\lambda, \mu} \mathfrak{A}_{\lambda} \widetilde{\otimes} \mathfrak{B}_{\mu}$. For the projective tensor product of bornological spaces (and of bornological algebras) see [12, §2.1.2]. In the next theorem and elsewhere we write $K V$ for Karoubi-Villamayor's $K$-theory.

Theorem 8.1 Let $S$ be one of $\ell^{p}, \ell^{p+}(0<p<\infty)$ or $\ell^{p-}(0<p \leq \infty)$.

i) The functor BAlg $\rightarrow \mathfrak{A b}, \mathfrak{A} \mapsto K H_{*}\left(I_{\ell^{1}(\mathfrak{A})}\right)$ is Hölder homotopy invariant and we have $K H_{*}\left(I_{S(\mathfrak{A})}\right)=K H_{*}\left(I_{\ell^{1}(\mathfrak{A})}\right)$ for all $S$ as above.

ii) For every bornological algebra $\mathfrak{A}$

$$
K H_{n}\left(I_{\ell^{1}(\mathfrak{A})}\right)= \begin{cases}K V_{n}\left(I_{\ell^{1}(\mathfrak{A})}\right) & n \geq 1 \\ K_{n}\left(I_{\ell^{1}(\mathfrak{A})}\right) & n \leq 0 .\end{cases}
$$

iii) If $\mathfrak{A}$ is a local Banach algebra and $n \geq 0$, then there is a natural split monomorphism $K_{n}^{\text {top }}(\mathfrak{A}) \rightarrow K H_{n}\left(I_{\ell^{1}(\mathfrak{A})}\right)$.

Proof Recall that $K H$ satisfies excision, vanishes on nilpotent rings and commutes with filtering colimits [23]. On the other hand, $\ell^{q}(\mathfrak{A}) / \ell^{p}(\mathfrak{A})$ is nilpotent for $p<q<\infty$ and

$$
\ell^{r-}(\mathfrak{A})=\operatorname{colim}_{s<r} \ell^{s}(\mathfrak{A}) \quad(0<r \leq \infty)
$$

It follows that $K H_{*}\left(I_{S(\mathfrak{A})}\right)=K H_{*}\left(I_{\ell^{1}(\mathfrak{A})}\right)$ for all $S$ as in the theorem. Recall also that $K H$ is $M_{2}$-stable. Hence $K H_{*}\left(I_{\ell^{1}(-)}\right)=K H_{*}\left(I_{\ell^{p}(-)}\right)$ is Hölder-homotopy invariant, by Theorem 7.8. This proves i). By [23, Proposition 1.5] (see also [5, Proposition 
5.2.3]), in order to prove ii) it suffices to show that $I_{\ell^{1}(\mathfrak{A})}$ is $K_{0}$-regular. By definition, a ring $A$ is $K_{0}$-regular if for each $n \geq 1$ the canonical map

$$
K_{0}(A) \rightarrow K_{0}\left(A\left[t_{1}, \ldots, t_{n}\right]\right)
$$

is an isomorphism. This is equivalent to the requirement that for $t=\left(t_{1}, \ldots, t_{n}\right)$, the map

$$
\epsilon: A[\underline{t}] \rightarrow A[\underline{t}], \epsilon(f)=f(0)
$$

induce an isomorphism in $K_{0}$. Observe that

$$
\begin{aligned}
I_{\ell^{1}(\mathfrak{A})}[\underline{t}] & =\left(\ell^{1}(\mathfrak{A}) \#_{\mathcal{P}} \Gamma\right)[\underline{t}] \\
& =\left(\ell^{1}(\mathfrak{A})[\underline{t}]\right) \#_{\mathcal{P}} \Gamma .
\end{aligned}
$$

Also note that, for the projective tensor product,

$$
\begin{aligned}
\ell^{1}\left(C^{\infty}([0,1], \mathfrak{A})\right) & =\ell^{1} \hat{\otimes} C^{\infty}([0,1], \mathbb{C}) \hat{\otimes} \mathfrak{A} \\
& =C^{\infty}\left([0,1], \ell^{1}(\mathfrak{A})\right) .
\end{aligned}
$$

Next we borrow an argument from [19, Proposition 3.4]. Consider the homomorphism

$$
\begin{aligned}
\phi: C^{\infty}\left([0,1], \ell^{1}(\mathfrak{A})\right)[\underline{t}] \rightarrow & C^{\infty}\left([0,1], \ell^{1}(\mathfrak{A})\right)[\underline{t}] \\
& \phi(f)(s, \underline{t})=f(s, s \underline{t}) .
\end{aligned}
$$

Using the identifications (8.1) and (8.2) we have a diagram

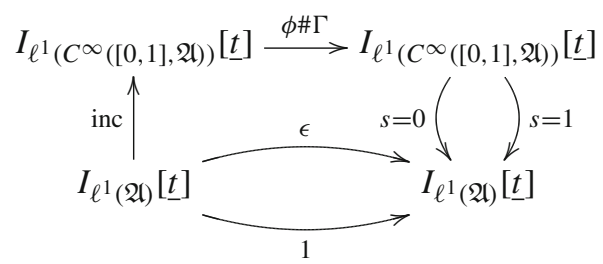

One checks that both the outer and the inner square commute. By Theorem 7.8, $K_{0}\left(\mathrm{ev}_{s=0} \# \Gamma\right)=K_{0}\left(\mathrm{ev}_{s=1} \# \Gamma\right)$. It follows that $K_{0}(\epsilon)$ is the identity; this proves ii). Next assume that $\mathfrak{A}$ is a local Banach algebra; then $K_{0}^{\text {top }}(\mathfrak{A})=K_{0}(\mathfrak{A})$. On the other hand, by the universal property of the crossed product, we have a map

$$
I_{\ell^{1}(\mathfrak{A})}=\left(\ell^{1} \hat{\otimes} \mathfrak{A}\right) \#_{\mathcal{P}} \Gamma \rightarrow \mathcal{L}^{1} \hat{\otimes} \mathfrak{A}
$$

Composing this map with the inclusion

$$
\mathfrak{A} \rightarrow I_{\ell^{1}(\mathfrak{A})}, a \mapsto a E_{1,1},
$$


we obtain the map

$$
\mathfrak{A} \rightarrow \mathcal{L}^{1} \hat{\otimes} \mathfrak{A}, \quad a \mapsto a \hat{\otimes} E_{1,1} .
$$

Since the latter map induces an isomorphism in $K_{0}$, it follows that (8.4) induces a split monomorphism $K_{0}(\mathfrak{A}) \rightarrow K_{0}\left(I_{\ell^{1}(\mathfrak{A})}\right)$. Thus we have established iii) for $n=0$. For the case $n \geq 1$, we consider the simplicial algebras of $C^{\infty}$ functions on the topological standard simplices and of polynomial functions on the algebraic standard simplices:

$$
\Delta^{\operatorname{dif}}:[n] \mapsto C^{\infty}\left(\Delta^{n}\right)
$$

and

$$
\Delta^{\mathrm{alg}}:[n] \mapsto \mathbb{C}\left[t_{0}, \ldots, t_{n}\right] /\left\langle\sum t_{i}-1\right\rangle
$$

Set

$$
\begin{aligned}
\Delta^{\operatorname{dif}} \mathfrak{A} & =\Delta^{\text {dif }} \hat{\otimes} \mathfrak{A} \text { and } \\
\Delta^{\text {alg }} \mathfrak{A} & =\Delta^{\text {alg }} \otimes_{\mathbb{C}} \mathfrak{A} .
\end{aligned}
$$

For $n \geq 1$, we have

$$
\begin{aligned}
& K_{n}^{\text {top }}(\mathfrak{A})=\pi_{n} B G L\left(\Delta^{\text {dif }} \mathfrak{A}\right), \\
& K V_{n}(\mathfrak{A})=\pi_{n} B G L\left(\Delta^{\text {alg }} \mathfrak{A}\right) .
\end{aligned}
$$

Hence for $K V(\mathfrak{A})=B G L\left(\Delta^{\operatorname{alg} \mathfrak{A}}\right)$, there is a map

$$
K_{n}^{\text {top }}(\mathfrak{A}) \rightarrow \pi_{n}\left(K V\left(\Delta^{\text {dif }}(\mathfrak{A})\right)\right)
$$

Composing the latter map with that induced by the inclusion (8.4), and using parts i) and ii), we get a homomorphism

$$
K_{n}^{\mathrm{top}}(\mathfrak{A}) \rightarrow \pi_{n} K V\left(I_{\ell^{1}\left(\Delta^{\mathrm{dif}} \mathfrak{A}\right)}\right) \cong K V_{n}\left(I_{\ell^{1}(\mathfrak{A})}\right)=K H_{n}\left(I_{\ell^{1}(\mathfrak{A})}\right)
$$

Composing (8.6) with the homomorphism induced by (8.3) we obtain

$$
K_{n}^{\mathrm{top}}(\mathfrak{A}) \rightarrow K H_{n}\left(\mathcal{L}^{1} \hat{\otimes} \mathfrak{A}\right)
$$

But by [9, Theorem 6.2.1] the comparison map

$$
K H_{n}\left(\mathcal{L}^{1} \hat{\otimes} \mathfrak{A}\right) \rightarrow K_{n}^{\text {top }}\left(\mathcal{L}^{1} \hat{\otimes} \mathfrak{A}\right)
$$

is an isomorphism. One checks that the latter map composed with (8.7) is equivalent to that induced by (8.5). But (8.5) induces an isomorphism in $K^{\text {top }}$ of local Banach algebras. This proves that (8.6) is a split monomorphism, concluding the proof. 
Theorem 8.2 i) The functor BAlg $\rightarrow \mathfrak{A} \mathfrak{b}, \mathfrak{A} \mapsto K H_{*}\left(I_{c_{0}(\mathfrak{A})}\right)$ is invariant under continuous homotopies.

ii) For every bornological algebra $\mathfrak{A}$

$$
K H_{n}\left(I_{c_{0}(\mathfrak{A})}\right)= \begin{cases}K V_{n}\left(I_{c_{0}(\mathfrak{A})}\right) & n \geq 1 \\ K_{n}\left(I_{c_{0}(\mathfrak{A})}\right) & n \leq 0 .\end{cases}
$$

iii) If $\mathfrak{A}$ is a bornolocal $C^{*}$-algebra and $n \geq 0$, then there is a natural split monomorphism $K_{n}^{\text {top }}(\mathfrak{A}) \rightarrow K H_{n}\left(I_{c_{0}(\mathfrak{A})}\right)$.

Proof As in Theorem 8.1, part i) follows from Theorem (7.8). To prove part ii), first observe that

$$
\begin{aligned}
c_{0}(C([0,1], \mathfrak{A})) & =C_{0}(\mathbb{N}, C([0,1], \mathfrak{A})) \\
& =C\left([0,1], c_{0}(\mathfrak{A})\right) .
\end{aligned}
$$

Then use the argument of the proof of part ii) of Theorem 8.1. To prove part iii) first observe that if $\mathfrak{A}$ is a bornolocal $C^{*}$-algebra, then for the spatial tensor product,

$$
c_{0}(\mathfrak{A})=c_{0} \tilde{\otimes} \mathfrak{A}
$$

Hence if $\mathcal{K}=\mathcal{K}\left(\ell^{2}(\mathbb{N})\right)$ is the $C^{*}$-algebra of compact operators then the map $\mathfrak{A} \rightarrow$ $\mathfrak{A} \otimes \mathcal{K}, a \rightarrow a \otimes E_{1,1}$ factors through $I_{c_{0}(\mathfrak{A})}$. Taking this into account, using the fact that, by [21, Theorem 10.9] and [19, Proposition 3.4], the comparison map $K H_{*}(\mathfrak{A} \tilde{\otimes}$ $\mathcal{K}) \rightarrow K_{*}^{\text {top }}(\mathfrak{A} \otimes \mathcal{K})$ is an isomorphism, and substituting continuous functions for $C^{\infty}$ functions, we may now proceed as in the prooof of part iii) of Theorem 8.1.

Remark 8.3 The argument of the proofs of part iii) of Theorems 8.1 and 8.2 does not work for $n<0$. Indeed, $K_{n}$ and $K_{n}^{\text {top }}$ do not agree for such $n$, not even on algebras on which the former is homotopy invariant. For example negative $K$-theory is homotopy invariant on commutative $C^{*}$-algebras [10, Theorem 1.2] yet $K_{n}(\mathbb{C})=0$ for $n<0$, while $K_{2 m}^{\text {top }}(\mathbb{C})=\mathbb{Z}$ for $m \in \mathbb{Z}$.

Remark 8.4 The argument of the proof of Theorem 8.1 shows that if $\mathfrak{A}$ is a local Banach algebra then $\mathfrak{A} \rightarrow \mathfrak{A} \hat{\otimes} \mathcal{L}^{1}$ factors through $I_{\ell^{1}(\mathfrak{A})}$ and the map

$$
K H_{n}\left(I_{\ell^{1} \mathfrak{A}}\right) \rightarrow K H_{n}\left(\mathfrak{A} \hat{\otimes} \mathcal{L}^{1}\right)=K_{n}^{\text {top }}(\mathfrak{A})
$$

is onto for $n \geq 0$. Similarly, the argument of the proof of 8.2 shows that for $\mathfrak{A}$ a bornolocal $C^{*}$-algebra the map $\mathfrak{A} \rightarrow \mathfrak{A} \otimes \mathcal{K}$ factors through $I_{c_{0}(\mathfrak{A})}$ and

$$
K H_{n}\left(I_{c_{0}(\mathfrak{A})}\right) \rightarrow K H_{n}(\mathfrak{A} \widetilde{\otimes} \mathcal{K})=K_{n}^{\text {top }}(\mathfrak{A})
$$

is onto for $n \geq 0$. 
$8.2 \mathrm{~K}$-theory and cyclic homology

Theorem 8.5 Let $\mathfrak{A}$ be a bornological algebra and let $S$ be $c_{0}, \ell^{p}, \ell^{p+}(0<p<\infty)$, or $\ell^{p-}(0<p \leq \infty)$. Then there are long exact sequences $(n \in \mathbb{Z})$

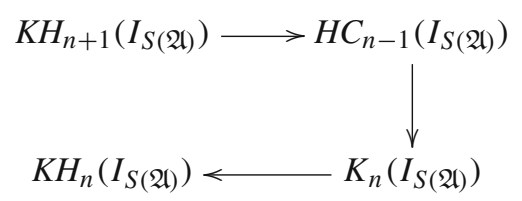

and

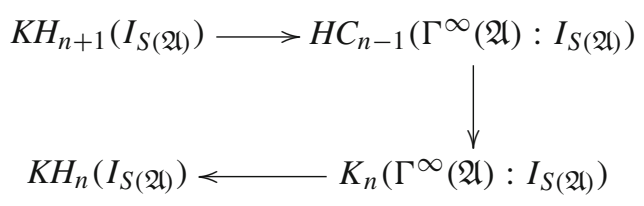

Proof Let $K^{\text {nil }}=$ hofi $(K \rightarrow K H)$ be the homotopy fiber of the comparison map. By [5, diagram (86)], there is a natural map $v: K^{\text {nil }}(A) \rightarrow H C(A)[-1]$, defined for every $\mathbb{Q}$-algebra $A$. Write $K^{\text {ninf }}=\operatorname{hofi}(v)$; by [7, Proposition 8.2.4] $K^{\text {ninf }}$ is excisive, $M_{2}$ stable and nilinvariant, and $K_{*}^{\text {ninf }}$ commutes with filtering colimits. Hence to prove the theorem it suffices to show that

$$
K_{*}^{\operatorname{ninf}}\left(I_{S(\mathfrak{A})}\right)=0
$$

Note also that if $S \neq c_{0}$, then

$$
K_{*}^{\operatorname{ninf}}\left(I_{S(\mathfrak{A})}\right)=K_{*}^{\operatorname{ninf}}\left(I_{\ell^{1}(\mathfrak{A})}\right)
$$

by the same argument as that used in the proof of Theorem 8.1 to prove the analogous assertion for $K H$. Thus we may assume from now on that $S \in\left\{c_{0}, \ell^{1}\right\}$. By [9, Proposition 3.1.4], to prove (8.10) it suffices to show that $I_{S(\mathfrak{A})}$ is $K^{\text {inf }}$-regular. Here $K^{\text {inf }}$ is infinitesimal $K$-theory; by [4] it is excisive and $M_{2}$-stable. Hence, the same argument as that used in the proof of Theorems 8.1 and 8.2 to prove that $I_{S(\mathfrak{A})}$ is $K_{0}$-regular applies to show that it is also $K^{\mathrm{inf}}$-regular. This completes the proof.

Remark 8.6 By Examples 5.3, we have

$$
K H_{*}\left(\Gamma^{\infty}(\mathfrak{A})\right)=H C_{*}\left(\Gamma^{\infty}(\mathfrak{A})\right)=K_{*}\left(\Gamma^{\infty}(\mathfrak{A})\right)=0
$$

for unital $\mathfrak{A}$. Hence in the unital case, the second sequence of Theorem 8.5 can be equivalently expressed in terms of the quotient $\Gamma^{\infty}(\mathfrak{A}) / I_{S(\mathfrak{A})}$; we have a long exact sequence 


$$
\begin{gathered}
K H_{n+1}\left(\Gamma^{\infty}(\mathfrak{A}) / I_{S(\mathfrak{A})}\right) \longrightarrow H C_{n-1}\left(\Gamma^{\infty}(\mathfrak{A}) / I_{S(\mathfrak{A})}\right) \\
\qquad H_{n}\left(\Gamma^{\infty}(\mathfrak{A}) / I_{S(\mathfrak{A})}\right) \longleftrightarrow K_{n}\left(\Gamma^{\infty}(\mathfrak{A}) / I_{S(\mathfrak{A})}\right)
\end{gathered}
$$

Acknowledgments Most of the research for this paper was carried out during visits of B. Abadie to the Universidad de Buenos Aires and of G. Cortiñas to the Universidad de la República. We are thankful to these institutions for their hospitality. G. Cortiñas wishes to thank his colleague Daniel Carando for useful discussions about topological tensor products and Ruy Exel for many useful discussions and for patiently explaining his paper [15].

\section{References}

1. Buss, A., Exel, R.: Fell bundles over inverse semigroups and twisted étale groupoids. J. Oper. Theory 67(1), 153-205 (2012)

2. Calkin, J.W.: Two-sided ideals and congruences in the ring of bounded operators in Hilbert space. Ann. Math. 42(2), 839-873 (1941)

3. Cohn, P.M.: Some remarks on the invariant basis property. Topology 5, 215-228 (1966)

4. Cortiñas, G.: The obstruction to excision in K-theory and in cyclic homology. Invent. Math. 164(1), 143-173 (2006)

5. Cortiñas, G.: Algebraic v. topological K-theory: a friendly match. In: Topics in Algebraic and Topological K-Theory. Lecture Notes in Mathematics, vol. 2011, pp. 103-165. Springer, Berlin (2008)

6. Cortiñas, G.: Cyclic Homology, Tight Crossed Products, and Small Stabilizations. Available at arXiv: 1304.3508

7. Cortiñas, G., Ellis, E.: Isomorphism conjectures with proper coefficients. Available at arXiv:1108.5196v3.

8. Cortiñas, G.: Bivariant algebraic K-theory. J. Reine Angew. Math. 610, 71-123 (2007)

9. Cortiñas, G., Thom, A.: Comparison between algebraic and topological K-theory of locally convex algebras. Adv. Math. 218(1), 266-307 (2008)

10. Cortiñas, G., Thom, A.: Algebraic geometry of topological spaces I. Acta. Mathematica. 209(1), 83131 (2012)

11. Cuntz, J.: K-theory for certain C*-algebras. Ann. Math. (2) 113(1), 181-197 (1981). doi:10.2307/ 1971137. MR 604046 (84c:46058)

12. Cuntz, J., Meyer, R., Rosenberg, J.M.: Topological and bivariant K-theory. Oberwolfach Seminars. Birkhäuser Verlag, Basel (2007)

13. Cuntz, J., Quillen, D.: Excision in bivariant periodic cyclic cohomology. Invent. Math. 127(1), 67-98 (1997)

14. Dykema, K., Figiel, T., Weiss, G., Wodzicki, M.: Commutator structure of operator ideals. Adv. Math. 185(1), 1-79 (2004)

15. Exel, R.: Inverse semigroups and combinatorial $C^{*}$-algebras. Bull. Braz. Math. Soc. (N.S.) 39(2), 191-313 (2008)

16. Garling, D.J.H.: On ideals of operators in Hilbert space. Proc. Lond. Math. Soc. 3(1), 115-138 (1967)

17. Higson, N.: Algebraic K-theory of stable $C^{*}$-algebras. Adv. Math. 67(1), 140 (1988)

18. Karoubi, M., Villamayor, O.: K -théorie algébrique et K -théorie topologique. I. Math. Scand. 28(1971), 265-307 (1972)

19. Rosenberg, J.: Comparison between algebraic and topological K-theory for Banach algebras and $\mathrm{C}^{*}$-algebras. In: Handbook of K-Theory, vol. 1, 2, pp. 843-874. Springer, Berlin (2005)

20. Simon, B.: Trace ideals and their applications, 2nd edn. Mathematical Surveys and Monographs, vol. 120. AMS, Providence (2005)

21. Suslin, A.A., Wodzicki, M.: Excision in algebraic K-theory. Ann. Math. (2) 136(1), 51-122 (1992)

22. Wagoner, J.B.: Delooping classifying spaces in algebraic K-theory. Topology 11, 349-370 (1972)

23. Weibel, C.A.: Homotopy algebraic K-theory. Algebraic K-theory and algebraic number theory (Honolulu, HI, 1987). In: Contemp. Math., vol. 83, pp. 461-488. American Mathematical Society, Providence (1989)

24. Wodzicki, M.: Algebraic K-theory and functional analysis. In: First European Congress of Mathematics, vol. II (Paris, 1992), pp. 485-496. Progr. Math., vol. 120. Birkhäuser, Basel (1994) 